\title{
Spatial resolution and dissipation rate estimation in Taylor-Couette flow for tomographic PIV
}

\author{
S. Tokgoz $\cdot$ G. E. Elsinga $\cdot$ R. Delfos $\cdot$ \\ J. Westerweel
}

Received: 5 October 2011/Revised: 12 March 2012/Accepted: 24 April 2012/Published online: 15 May 2012

(C) The Author(s) 2012. This article is published with open access at Springerlink.com

\begin{abstract}
This paper assesses the spatial resolution and accuracy of tomographic particle image velocimetry (PIV). In tomographic PIV the number of velocity vectors are of the order of the number of reconstructed particle images, and sometimes even exceeds this number when a high overlap fraction between adjacent interrogations is used. This raises the question of the actual spatial resolution of tomographic PIV in relation to the various flow scales. We use a Taylor-Couette flow of a fluid between two independently rotating cylinders and consider three flow regimes: laminar flow, Taylor vortex flow and fully turbulent flow. The laminar flow has no flow structures, and the measurement results are used to assess the measurement uncertainty and to validate the accuracy of the technique for measurements through the curved wall. In the Taylor vortex flow regime, the flow contains large-scale flow structures that are much larger than the size of the interrogation volumes and are fully resolved. The turbulent flow regime contains a range of flow scales. Measurements in the turbulent flow regime are carried out for a Reynolds number $R e$ between 3,800 and 47,000. We use the measured torque on the cylinders to obtain an independent estimate of the energy dissipation rate and estimate of the Kolmogorov length scale. The data obtained by tomographic PIV are assessed by estimating the dissipation rate and comparing the result against the dissipation rate
\end{abstract}

S. Tokgoz $(\bowtie)$

LaVision GmbH, Anna-Vandenhoeck-Ring 19,

37081 Gottingen, Germany

e-mail: s.tokgoez@tudelft.nl

S. Tokgoz - G. E. Elsinga - R. Delfos - J. Westerweel

Laboratory for Aero and Hydrodynamics,

Department of Mechanical Engineering,

Delft University of Technology, Delft, The Netherlands obtained from the measured torque. The turbulent flow data are evaluated for different sizes of the interrogation volumes and for different overlap ratios between adjacent interrogation locations. The results indicate that the turbulent flow measurements for the lowest $R e$ could be (nearly) fully resolved. At the highest $R e$ only a small fraction of the dissipation rate is resolved, still a reasonable estimate of the total dissipation rate could be obtained by means of using a sub-grid turbulence model. The resolution of tomographic PIV in these measurements is determined by the size of the interrogation volume. We propose a range of vector spacing for fully resolving the turbulent flow scales. It is noted that the use of a high overlap ratio, that is, $75 \%$, yields a substantial improvement for the estimation of the dissipation rate in comparison with data for 0 and $50 \%$ overlap. This indicates that additional information on small-scale velocity gradients can be obtained by reducing the data spacing.

\section{Introduction}

The development of modern multi-camera methods, such as tomographic particle image velocimetry (tomographic PIV; Elsinga et al. 2006), makes it possible to measure all three components and their spatial derivatives of the instantaneous velocity field in a volumetric domain. Such data enable the investigation of the instantaneous structure of turbulent flows, and they provide quantitative experimental data on the full deformation tensor and derived quantities, such as the energy dissipation rate. For turbulence measurements, it is necessary to resolve the spatial velocity gradients, which requires a high spatial resolution. However, the spatial resolution in tomographic PIV is limited by the maximum density of particle images that can 
be recorded (Elsinga et al. 2006; Adrian and Westerweel 2011). As a consequence, there exists an upper limit to the number of reconstructed particle images. This is further augmented by the common practice to use overlapping interrogation domains, which may result in a final data density (determined by the total number of interrogation locations) that exceeds the estimated tracer particle density in the measurement volume. Hence, the question arises what the actual spatial resolution is of a tomographic PIV measurement. To validate the spatial resolution of tomographic PIV, we consider the (turbulent) dissipation rate in Taylor-Couette (TC) flow. For this flow, the dissipation rate is proportional to the torque applied to the rotating cylinders (Racina and Kind 2006). Hence, we have an independent measurement for the dissipation rate, and this can be compared against the dissipation rate estimated from the velocity data. A discrepancy between these values is a measure of the accuracy and the spatial resolution of the measurement, as the dissipation rate is determined by the smallest scales that appear in the flow.

A further challenge that is addressed in this paper lies in the fact that tomographic PIV is applied to a flow domain with a curved and moving outer wall, which complicates the measurement. Since tomographic PIV relies on the precise volumetric reconstruction of the scattering sites in the measurement volume, optical aberrations that are not accounted for in the calibration can deteriorate the quality of the reconstruction. The reconstruction and a volumetric self-calibration can be applied to correct for small optical distortions and aberrations.

Following the same categorisation as Andereck et al. (1986), we consider three Taylor-Couette flow regimes in this paper, namely laminar flow, flow with Taylor vortices and fully turbulent (i.e., "featureless" turbulent) flow. These regimes have increasing dissipation rates, that is, decreasing length scales. For the laminar flow case, we only have one dominant velocity gradient determined by the differential angular speed of the cylinders and the gap width between the cylinders. In this case, the flow can be fully resolved due to the absence of any small-scale variations of the velocity. In the case of the Taylor-vortex flow regime, we find large-scale vortical structures. Also here, by absence of small-scale motions, the measurement should be able to fully resolve the flow. The fully turbulent flow regime contains small-scale flow structures. The flow is fully three-dimensional and the turbulent kinetic energy is dissipated in small-scale vortices. The scale of these vortices depends on the Reynolds number. By increasing the Reynolds number, we can decrease the smallest flow scales relative to the measurement resolution.

In order to quantify the spatial resolution of tomographic PIV, we compare the computed dissipation rate values with the dissipation rates that were estimated using torque measurements. We discuss the effect of the Reynolds number, the interrogation window size and the data spacing relative to the interrogation window size (i.e., window overlap) on the spatial resolution of tomographic PIV. Additionally, we also use the large eddy PIV method (Sheng et al. 2000) to estimate the dissipation rate and discuss its performance.

An outline of this paper is as follows. A brief literature review is given in Sect. 2. The implementation of tomographic PIV for a Taylor-Couette flow system is discussed in Sect. 3. We briefly explain several problems that were encountered during the implementation. The validation of the experimental method is done for the analytically welldefined laminar flow case, which is basically a stable circular Couette flow, are given in Sect. 4.1. The effect of a curved and rotating outer cylinder between the flow domain and the cameras on the measurement results is tested in the same section. Then, in Sects. 4.2 and 4.3, the characteristics of Taylor vortex flow and the fully turbulent flow regimes are analysed. Estimates of the dissipation rate and the effect of interrogation window size relative to the Kolmogorov length scale are given in Sect. 5. The main findings are summarised in Sect. 6.

\section{Background}

Taylor-Couette systems basically consist of two independently rotating concentric cylinders. Isaac Newton is believed to be one of the first scientists attracted to the flow between these rotating cylinders (Donnelly 1991).

The advantage of the Taylor-Couette setup is the possibility to examine the flow stability in a small closed environment, which can be manipulated simply by adjusting the rotation speeds of the cylinders. In addition, usage of a transparent outer cylinder makes it possible to observe the elementary flow characteristics with different visualisation techniques (Di Prima and Swinney 1985). An extensive characterisation of flow regimes in TaylorCouette systems, based on flow visualisation analysis, was reported by Andereck et al. (1986), which is regarded as a reference for defining the flow patterns in Taylor-Couette flows.

The set of parameters used to describe the TaylorCouette flow varied over the years. In this paper, we adopt the parameters to characterise the Taylor-Couette flow as defined by Dubrulle et al. (2005). The Reynolds numbers for inner cylinder and outer cylinder, based on the gap $d$, are defined as $\operatorname{Re}_{i}=\left(r_{i} \Omega_{i} d / v\right)$ and $\operatorname{Re}_{o}=\left(r_{o} \Omega_{o} d / v\right)$, respectively. $\Omega_{i}$ and $\Omega_{o}$ represent the angular velocities of the inner and the outer cylinders, and $v$ represents the kinematic viscosity of the fluid. The Rotation number $(R o)$ and the shear Reynolds number $\left(R e_{s}\right)$ are defined as: 
Table 1 Flow conditions of the laminar (LF), Taylor vortex flow (TVF) and fully turbulent flow (FT)

\begin{tabular}{|c|c|c|c|c|c|c|c|c|c|c|}
\hline Flow type & $R e_{i}(-)$ & $R e_{o}(-)$ & $R e_{s}(-)$ & $\operatorname{Ro}(-)$ & $\Omega_{i}(\mathrm{rad} / \mathrm{s})$ & $\Omega_{o}(\mathrm{rad} / \mathrm{s})$ & $\Delta t(\mathrm{~ms})$ & Rec. rate $(\mathrm{Hz})$ & Number of vectors & $\delta_{x}(\mathrm{~mm})$ \\
\hline LF & - & 643 & 615 & 0.091 & - & 0.48 & 10.0 & 4.7 & $107 \times 61 \times 28$ & 0.370 \\
\hline TVF & 1,000 & 500 & 565 & -0.231 & 0.88 & 0.38 & 12.5 & 4.7 & $92 \times 61 \times 28$ & 0.370 \\
\hline FT4700 & 1,850 & $-2,900$ & 4,700 & 0.019 & 1.57 & -2.26 & 2.5 & 4.7 & $92 \times 61 \times 28$ & 0.370 \\
\hline FT3800 & 1,900 & $-1,900$ & 3,800 & 0 & 1.63 & -1.51 & 5.0 & 7.55 & $107 \times 55 \times 26$ & 0.391 \\
\hline FT6200 & 3,100 & $-3,100$ & 6,200 & 0 & 2.70 & -2.45 & 5.0 & 7.55 & $107 \times 55 \times 26$ & 0.391 \\
\hline FT11000 & 5,500 & $-5,500$ & 11,000 & 0 & 4.78 & -4.39 & 3.0 & 7.55 & $107 \times 55 \times 26$ & 0.391 \\
\hline FT14000 & 7,000 & $-7,000$ & 14,000 & 0 & 6.09 & -5.59 & 2.0 & 7.55 & $107 \times 55 \times 26$ & 0.391 \\
\hline FT17000 & 8,500 & $-8,500$ & 17,000 & 0 & 7.41 & -6.79 & 1.5 & 7.55 & $107 \times 55 \times 26$ & 0.391 \\
\hline FT29000 & 14,500 & $-14,500$ & 29,000 & 0 & 12.63 & -11.56 & 1.0 & 7.55 & $107 \times 55 \times 26$ & 0.391 \\
\hline FT36000 & 18,000 & $-18,000$ & 36,000 & 0 & 15.65 & -14.33 & 0.5 & 7.55 & $107 \times 55 \times 26$ & 0.391 \\
\hline FT47000 & 23,500 & $-23,500$ & 47,000 & 0 & 20.42 & -18.72 & 0.25 & 7.55 & $107 \times 55 \times 26$ & 0.391 \\
\hline
\end{tabular}

Parameters of the tomographic PIV measurements for the flow conditions are given on the last four columns. $\Delta t$ is the exposure time delay. The number of vectors are given in the $x, y$ and $z$ directions, respectively (see Figs. 1 and 2); $\delta_{x}$ stands for the distance between the vectors. The number of vectors and $\delta_{x}$ values are given for computations with $40 \times 40 \times 40$ voxel $^{3}$ final interrogation windows with a $75 \%$ overlap

$R o=(1-\eta) \frac{R e_{i}+R e_{o}}{\eta R e_{o}-R e_{i}}$,

$R e_{s}=\frac{2\left|\eta R e_{o}-R e_{i}\right|}{1+\eta}$

where $\eta=r_{i} / r_{o}$ is the gap ratio. The experimental parameters for all measurements are summarised in Table 1.

So far, field-based experimental studies mainly focused on 2D structures of the flow because of the limited capabilities of available experimental methods. Wereley and Lueptow $(1998,1999)$ performed 2D PIV measurements in Taylor-Couette flow. However, they could only measure the axial and radial components of the flow velocity. They applied a glass box, filled with a liquid that matches the refractive index of the working fluid, that encloses the Taylor-Couette flow system in order to avoid effects due to refraction from the curved outer cylinder wall. Since then, 2D PIV has been used to examine different flow characteristics of Taylor-Couette flows (Akonur and Lueptow 2003; Wang et al. 2005; Smieszek and Egbers 2005; Racina and Kind 2006; Abcha et al. 2008; Deng et al. 2009). Akonur and Lueptow (2003) performed planar PIV in radial-azimuthal planes in a setup very similar to the one of Wereley and Lueptow (1998). In order to obtain the third component of the velocity, they combined their results with those of Wereley and Lueptow (1998), which were in the axial-radial direction. With the help of phase averaging, they obtained time-resolved, three-dimensional and threecomponent PIV results. So far, their work has been the only experimental attempt to analyse volumetric flow structures in a Taylor-Couette system by means of PIV. Recently, Ravelet et al. (2010) applied Stereo PIV to Taylor-Couette flow for the first time. They performed measurements in the axial-radial plane, where the azimuthal velocity is in the out-of-plane direction. They also performed torque measurements on the inner cylinder. The combination of Stereo PIV and torque measurements was used to explore the torque scaling in relation to the flow field.

Despite several papers on the application of PIV to Taylor-Couette flows, the reliability of PIV measurements in Taylor-Couette flow has not been studied widely. Akonur and Lueptow (2003) report an error for PIV measurements of laminar flow to be $1 \%$ for azimuthal and $4 \%$ for radial velocities, relative to the inner cylinder velocity. On the other hand, Ravelet et al. (2010) showed the error level does not exceed $1 \%$ for the same components, using stereoscopic PIV measurements. However, they report a significant velocity difference in regions close to the outer cylinder walls. This may be attributed to refraction effects due to the curved cylinder walls.

According to the energy-cascade model, the turbulent energy is dissipated on the smallest eddies, and it is important to estimate the dissipation rate for some industrial processes such as mixing (Jiménez et al. 1993; Saarenrinne and Piirto 2000; Sharp and Adrian 2001). The approach to determine the local dissipation rate from tomographic PIV data follows those reported by others, using planar PIV data (Sheng et al. 2000; Sharp and Adrian 2001; Baldi and Yianneskis 2003; Racina and Kind 2006; Tanaka and Eaton 2007; Lavoie et al. 2007). In order to resolve the smallest scales in turbulence and to capture the velocity gradients accurately, measurements of turbulent flows should ideally have a resolution of the order of the Kolmogorov microscale (Sharp and Adrian 2001; Adrian and Westerweel 2011). Nevertheless, for accurate results, the knowledge of velocity gradients in all directions, which is not possible by 2D PIV, is required (Adrian and Westerweel 2011). The missing data can be estimated by assuming local isotropy or by making use of symmetry properties in the statistics of the local deformation tensor 
(Sharp et al. 2000; Sharp and Adrian 2001; Racina and Kind 2006). Those assumptions limit the calculations because of significant non-isotropic and inhomogeneous structure of the flow (Sharp et al. 2000). Sharp and Adrian (2001) showed that, in case of additional correction methods, success of measuring the dissipation rate via PIV increases up to $70 \%$ of the actual dissipation rate. Racina and Kind (2006) followed the same assumptions. They used 2D PIV measurements to calculate the dissipation rate in a Taylor-Couette system for the first time. Independent torque measurements allowed them to compare the dissipation rate measurements with their actual values. The energy dissipation rate showed good reproducibility according to their results.

Recently, Worth et al. (2010) performed simulations and compared them with experiments in order to discuss the resolution of $2 \mathrm{D}$ and of tomographic PIV. They used errors as indicator of the effect of the noise and the spatial resolution over the velocity and the dissipation rates. In this paper, instead of simulations, we use torque measurements to validate the dissipation rates estimated from the PIV data.

\section{Experimental setup}

The measurements were performed in the Taylor-Couette setup at the Laboratory for Aero \& Hydrodynamics of the Delft University of Technology, which was used previously by Ravelet et al. (2010). It consists of two coaxial cylinders that can rotate independently. Additionally, the system allows performing torque measurement on the inner cylinder shaft. The radii of inner and outer cylinders are $r_{i}=110 \pm$ $0.05 \mathrm{~mm}$ and $r_{o}=120 \pm 0.05 \mathrm{~mm}$, respectively. This results in a gap of $d=r_{o}-r_{i}=10 \mathrm{~mm}$, and a corresponding gap ratio of $\eta=r_{i} / r_{o}=0.917$. The length of the inner cylinder is $L=220 \mathrm{~mm}$, which gives an axial aspect ratio of $\Gamma=L / d=22$. A sketch of the experimental setup is given in Fig. 1. The system is closed by top and bottom covers, which are rotating with the outer cylinder. The cylinders are transparent, allowing optical access. However, structural metal bars, which are placed inside of the inner cylinder, were found to cause strong reflections and noise on the recorded images. Therefore, another cylinder, which was painted black, was placed on the inside of the inner cylinder, to cover the structural bars. This improves the quality of the images considerably.

Velocity measurements were done using the tomographic PIV method (Elsinga et al. 2006). With tomographic PIV, it is possible to achieve a fully volumetric measurement of all three velocity components in the instantaneous flow field. The recording and the image

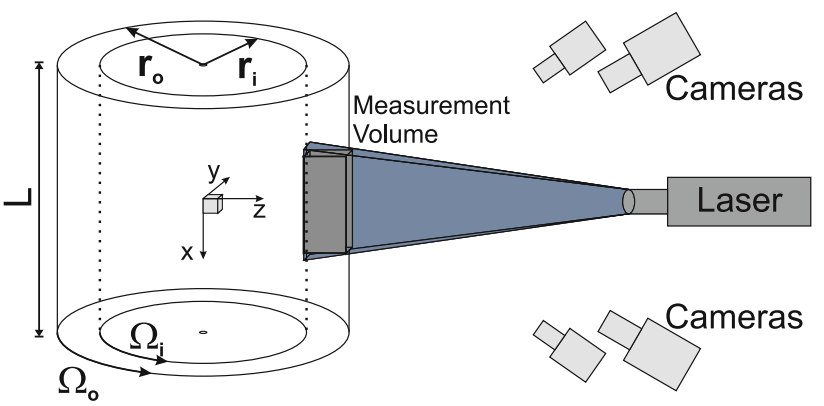

Fig. 1 Sketch of the experimental setup and definition of the coordinate system in the measurement volume; $x$ axial, $y$ azimuthal, and $z$ radial direction

analysis were done using commercial software (DAVIS by LaVision $\mathrm{GmbH}$ ). Four cameras (Imager Pro LX 16M) were used in double frame mode for recording particle images with a resolution of $4,800 \times 3,200$ pixels for laminar, Taylor vortex, and a fully turbulent flow case with $R e_{s}=4,700$ and $R o=0.019$. For the remaining fully turbulent flows $\left(R e_{s}=3,800-47,000\right.$, where $\left.R o=0\right)$, cameras with a resolution of 2,000 $\times 2,000$ pixels (Imager Pro X 4M) were used. Only about $1,000 \times 600$ pixels were used for all cases in order to achieve a higher image recording rate. Recording rate and laser pulse separation differ for each flow condition. Objectives with a $105 \mathrm{~mm}$ focal length were used, which were mounted on Scheimpflug adapters. In order to minimise the effect of the end gaps of the Taylor-Couette facility on the measurements, the images were recorded at the mid-height of the rotational axis of the Taylor-Couette setup (see Fig. 1). The dimensions of the volume recorded by all cameras is roughly $40 \times 20 \times 10 \mathrm{~mm}^{3}$ in axial, azimuthal and radial directions, respectively. One pixel in the recorded image corresponds to $37 \mu \mathrm{m}$ in flow field. The reconstructed volume size changes slightly between individual experiments.

It is convenient to interrogate the tomographic PIV data in a rectangular volume, although a cylindrical coordinate system is more appropriate for the Taylor-Couette geometry. In order to avoid interpolation errors in the conversion between coordinate systems, the Cartesian representation is followed throughout this paper.

The correspondence between the Cartesian and the cylindrical coordinate systems for the measurement volume is given in Fig. 2. Since the axial direction, $x$, is completely collinear in both coordinate systems, it is not shown in the figure. As shown, the $z$ and $r$ directions are collinear only in one axial-radial plane, where $\theta=0$. On the other hand, $y$ and $\theta$ directions are collinear on the same plane, as well. Hence, $x, y$ and $z$ components of the measured velocity data corresponds to axial, azimuthal and radial components of the velocities at the cylindrical coordinate system on the collinear plane. Thus, the 


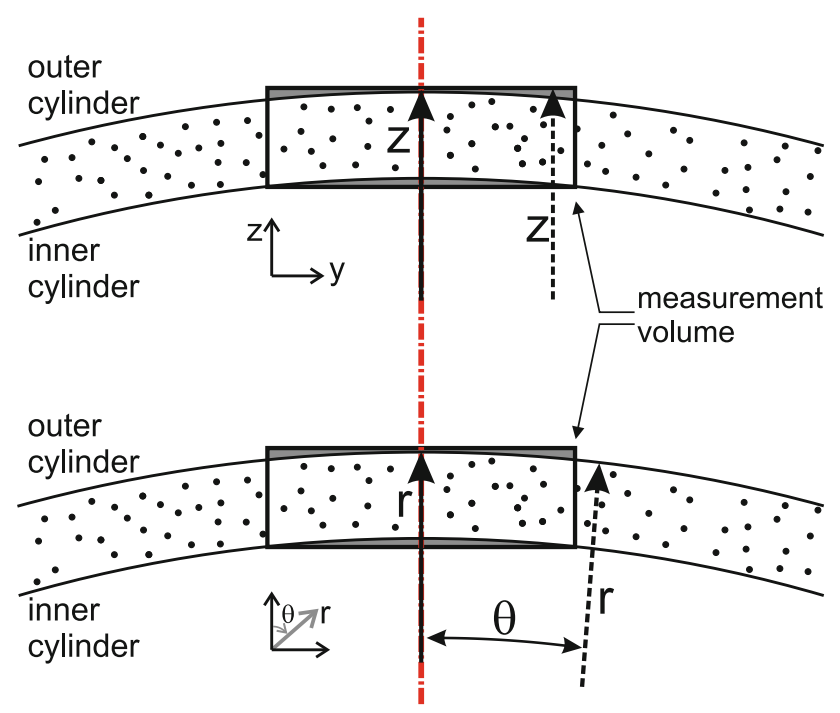

Fig. 2 Representation of the Cartesian (top) and cylindrical (bottom) coordinates for the experimental setup. Grey areas represent the zones, which are included to reconstructed volume, but are outside of the cylinders. Thus, they do not contain actual particles. Ghost particles appear in the gap between the cylinders as well as in the outside of the cylinders (grey areas)

$z$-coordinate and $r$-coordinate are interchangeable, whereas the $y$-coordinate and $\theta$-coordinate also coincide in this selected plane. Please note that all 2D plots in this paper are plotted on this collinear plane.

In order to avoid the effect of the reflections caused by volume illumination, we apply fluorescent (Fluostar) particles that contain Rhodamine B, with a mean diameter of $15 \mu \mathrm{m}$. These particles have a density of $1.1 \mathrm{~g} / \mathrm{cm}^{3}$. We applied optical $570 \mathrm{~nm}$ lowpass filters for rejecting the non-fluorescent illumination during the image acquisition. In order to have a homogeneous seeding distribution, the water including the seeding particles was mixed at high speeds of the inner and outer cylinders between two sets of recordings. Then, the system was stopped and the fluid was allowed to settle down. After that, the cylinders were taken to the desired rotational speeds, and PIV images were recorded after the flow reached a stationary state.

The seeding density is kept low in order to avoid speckle (Adrian and Westerweel 2011) and to achieve a high quality in the tomographic reconstruction (Elsinga et al. 2006). The quality of the tomographic reconstruction decreases with the increasing number of, so-called, ghost particles. A detailed discussion on ghost particles, their formation and their effects on the results were presented by Elsinga et al. (2006, 2011). The reconstruction quality is proportional to the signal-to-noise ratio (SNR) between the number of actual $\left(N_{p}\right)$ and the ghost particles $\left(N_{g}\right)$, which is given by $S N R=N_{p} / N_{g}$ (Worth et al. 2010; Elsinga et al. 2011).

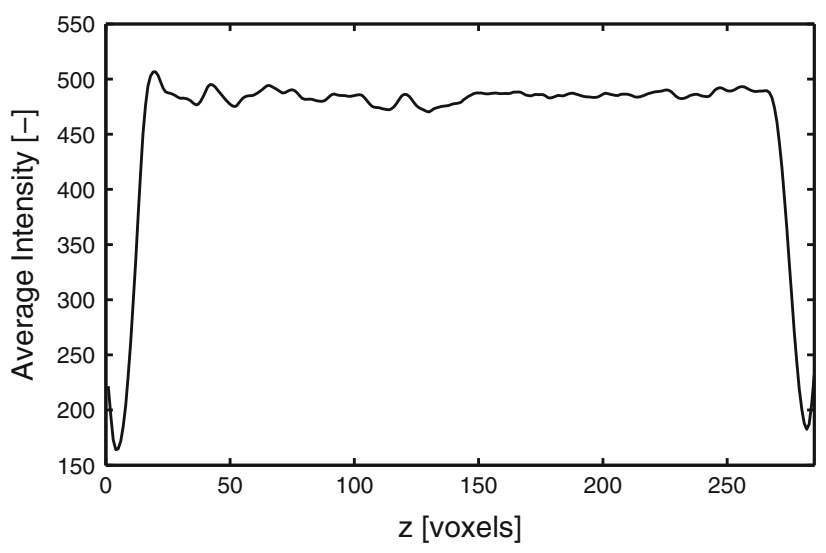

Fig. 3 Mean intensity profile along the $z$-direction (see Figs. 1 and 2) in the reconstructed measurement volume (over 150 instantaneous volumes). A width of $10 \mathrm{~mm}$ corresponds to 270 voxel units

At the same time, a high seeding density is desired to achieve better spatial resolution (Adrian and Westerweel 2011). Thus, a compromise should be found between reaching a higher spatial resolution and reducing the number of ghost particles. Based on these considerations and given the additional complexity of curved and moving walls, the seeding density was kept around the lower value of 0.025 'particles per pixel' (ppp) for the measurements presented here. This results in a SNR of 6.1, which is significantly above the minimum level of 2 that indicates a high-quality tomographic PIV measurement (Elsinga et al. 2011). The corresponding source density is $N_{S}=0.18$, which is sufficiently low to exclude speckle effects in the recorded images. The high quality of the tomographic reconstruction is also observable in the radial profile of the intensity distribution in the reconstructed volume (Fig. 3), which reveals the sharp contrast between the intensity inside and outside the liquid-filled gap.

The light source for illumination was a double-pulsed Nd:YAG laser (New Wave Solo-III) with $50 \mathrm{~mJ} /$ pulse energy at a wavelength of $532 \mathrm{~nm}$. We used optics with an anti-reflection coating consisting of two spherical lenses $(f=-50 \mathrm{~mm}, f=-40 \mathrm{~mm})$ and one cylindrical lens $(f=+200 \mathrm{~mm})$, which were placed between the laser and the test section to achieve the necessary dimensions of the laser beam for the illumination of the measurement volume.

In our Taylor-Couette system, it is not possible to directly control the temperature of the working fluid. However, the fluid and the ambient temperature were measured carefully between the recordings of each data set, and the angular velocities of the cylinders were adjusted to compensate for the temperature dependent fluid viscosity, so that we could maintain a constant flow Reynolds number. When the temperature difference between the beginning and the end of each set of recordings exceeds $0.5^{\circ} \mathrm{C}$, 
the data were considered invalid and were not used. Thus, variations in the temperature of the working fluid were less than $\pm 0.5^{\circ} \mathrm{C}$ for the results presented in this paper. The $\pm 0.5^{\circ} \mathrm{C}$ change in the temperature results in a maximum of $1.2 \%$ uncertainty in the kinematic viscosity of the fluid, which is water for the current study. Since each set of experiments takes around $20 \mathrm{~min}$, including the period to achieve stationary flow conditions, and given that the measurements were performed at relatively low angular velocities, this approach is assumed reliable.

\subsection{Calibration}

The procedure for the calibration of the camera system consists of two main steps. The first step is to determine the mapping of the recorded planes to all cameras, as it is usually done in Stereo PIV. This was done by traversing the calibration target in the $z$ direction along the gap width. The second step is the volumetric self-calibration method (Wieneke 2008) for refining the calibration.

Calibration of the camera system was done using a 1-mm thick, stainless steel, flat plate. The dimensions of the plate are $150 \times 20 \mathrm{~mm}^{2}$, where the short edge is placed tangential to the azimuthal flow direction. Circular holes with diameter of $0.4 \mathrm{~mm}$ were drilled. The distance between subsequent holes is $2.5 \mathrm{~mm}$ in both vertical and horizontal directions. At least 8 holes in all directions were present in each of the calibration image recordings. The calibration target was placed on a translating and rotating traversing mechanism, capable of positioning the target with micrometer precision. Due to the target thickness and the curvature of the cylinder, the calibration target can be translated only over $50 \%$ of the gap width. Thus, calibration images were recorded in three selected planes. The calibration for the remaining $50 \%$ of the gap was computed by extrapolating the calibration equation. During the calibration the gap between the cylinders was also filled with water.

The curved outer walls of the cylinders introduce some optical distortion. However, these distortions are small enough, so that they can be compensated for in the calibration. Since the tomographic reconstruction requires an error level better than 0.4 pixel (Elsinga et al. 2006) and the extrapolation of the mapping function can introduce further uncertainties, the volumetric self-calibration (Wieneke 2008) was applied for further refinement of the calibration. After several refinement steps with volumetric self-calibration, the maximum calibration error could be reduced from 0.329 to 0.019 pixel.

\subsection{Image processing}

Image processing was performed to reduce the effect of background noise and to increase the image quality of the recorded images. First, a sliding minimum intensity of $25 \times 25$ pixels was subtracted from all images to increase the signal-to-noise ratio. Then, a smoothing with a $3 \times 3$ pixel Gaussian kernel was applied. Tomographic reconstruction was performed with the MART algorithm (Elsinga et al. 2006). The intensity distribution averaged over 150 reconstructed volumes along the $z$ direction is given in Fig. 3. The distribution shows that the illuminated volume occurs for voxels located at $19 \leq z \leq 266$. Outside that region, the intensity values decrease approximately to one-third of that inside. The steep drop of the intensity indicates the presence of the cylinder walls. The comparison of intensities inside and outside of the cylinder walls reveals the contribution of ghost particles to the reconstructed volume (Elsinga et al. 2006), which appears to be $35 \%$ of the total intensity. The reconstructed volume size was around $40 \times 20 \times$ $10 \mathrm{~mm}^{3}$. This corresponds to a resolution of approximately 27 voxel $/ \mathrm{mm}$.

For the experiments of all flow types represented in this paper, the adaptive multi-pass approach was used for correlation. Unless stated otherwise (see Sects. 5.2.2, 5.2.3), the interrogation window size was $60 \times 60 \times 60$ voxels with a $50 \%$ overlap in the first pass and $40 \times 40 \times 40$ voxels with a $75 \%$ overlap in the final two passes. Spurious vectors were detected and removed by the universal outlier detection method (Westerweel and Scarano 2005). Linear interpolation was used to fill the gaps where the vectors were removed.

\section{Flow characteristics}

In this section, the accuracy of tomographic PIV for Taylor-Couette systems is initially validated using welldefined laminar flow. Then, the characteristics of the other flow regimes are given in terms of flow profiles, 3D visualisations, and coherent structures.

\subsection{Laminar flow and accuracy assessment}

The laminar flow case with only the outer cylinder rotating provides a steady flow that can be used to assess the accuracy of the tomographic PIV method.

Laminar Taylor-Couette flow is analytically well defined, with zero axial and radial velocities, and with an axisymmetric azimuthal velocity, $v$, given by (Dubrulle et al. 2005):

$v(r)=A r+\frac{B}{r}$,

where, $r$ is the radial distance with respect to the common axis of rotation, and $A$ and $B$ are constants, defined as: 


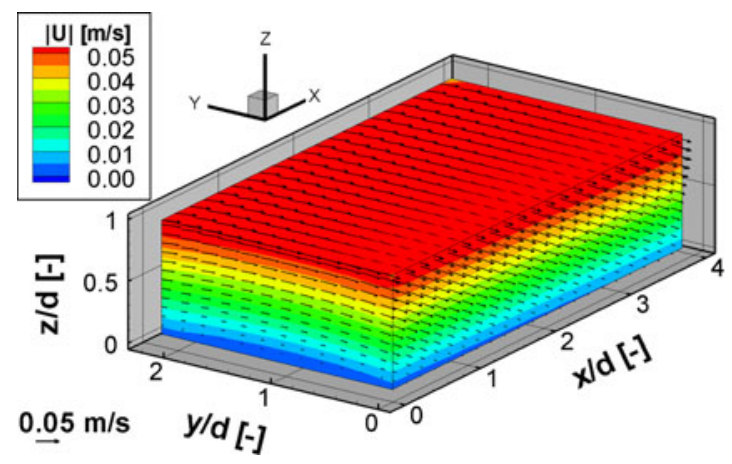

Fig. 4 3D plot (left), and 2D cross-section (right) of the laminar Taylor-Couette flow, obtained from 150 time-averaged instantaneous vector fields. Only every 5th vector in the $x$ and $y$ directions and every 2nd vector in the $z$ direction are shown. Color coding represents the absolute velocity $\left(|U|=\sqrt{u^{2}+v^{2}+w^{2}}\right)$. The data are represented in

$A=\frac{1}{1-\eta^{2}}\left(\Omega_{o}-\eta^{2} \Omega_{i}\right), \quad B=\frac{r_{i}^{2}}{1-\eta^{2}}\left(\Omega_{i}-\Omega_{o}\right)$,

where, $\Omega_{o}$ and $\Omega_{i}$ are the angular velocities of the outer and inner cylinders, respectively.

Laminar flow measurements were performed with only the outer cylinder rotating (i.e., $\Omega_{i}=0$ ). Corresponding Reynolds and rotation numbers are summarised in Table 1 . If we substitute $\Omega_{i}=0$, in (3-4):

$v(r)=\frac{\Omega_{0}}{1-\eta^{2}}\left(r-\frac{r_{i}^{2}}{r}\right)$.

The measured 3D velocity fields are shown in Fig. 4. The curved streamlines are in correspondence with the curvature of the cylinders.

Quantitative comparison between the analytical result and the measurements is necessary to assess the reliability of the method. The results are plotted in Fig. 5. Flow profiles presented here were obtained from an average over 150 instantaneous 3D vector fields. The profile of the azimuthal velocity $(v)$ is in good overall agreement with the analytical solution. The difference between the analytical solution and the tomographic PIV results does not exceed $3.2 \%$ of the outer cylinder velocity anywhere between the cylinders. Especially, in the region of $0.20 d \leq r-r_{i} \leq 0.50 d$ the deviation is below $2.5 \%$, and in the region $0.50 d \leq r-$ $r_{i} \leq 0.95 d$ it is below $1 \%$. Additionally, the maximum absolute values for the axial $(u)$ and the radial $(w)$ velocity components, which should be identical to zero, are within 0.7 and $0.5 \%$ of the outer cylinder velocity, respectively. Presence of non-zero axial and radial velocities can be explained as the result of the finite height of the experimental setup, which results in large-scale Ekman-like circulation (Dubrulle et al. 2005). Since the velocity deviation is always below $3.2 \%$, the effect of a moving and curved wall between the test section and the cameras appears not to significantly deteriorate the measurement quality.

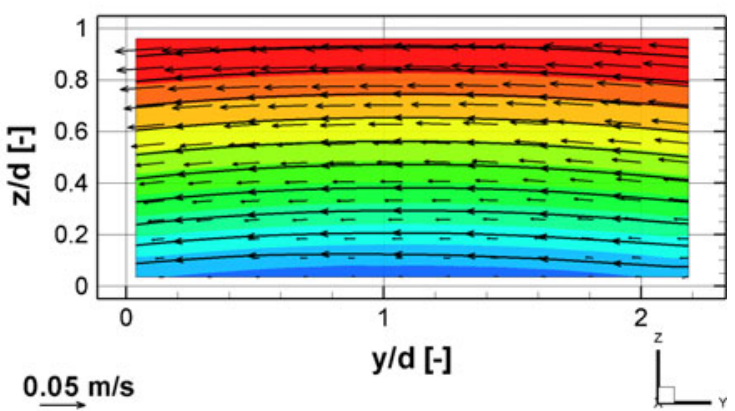

a Cartesian coordinate system (see Figs. 1 and 2), where $z=0$ corresponds to the inner cylinder surface, and $z=1$ corresponds to the outer cylinder surface. Both images are non-dimensionalised with the gap width $d$ between the cylinders

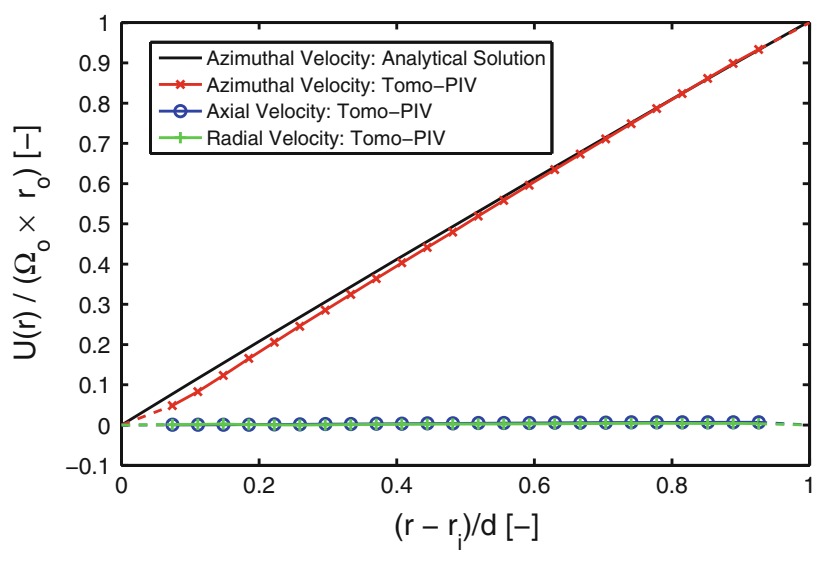

Fig. 5 Mean velocity of the laminar flow with only the outer cylinder rotating $\quad\left(\Omega_{o}=0.48 \mathrm{rad} / \mathrm{sec}, \Omega_{i}=0, R e_{o}=643, R o=0.091, R e_{s}=\right.$ $615)$, as a function of the radial distance. Time-averaging was performed over 150 instantaneous vector fields. All velocities are normalised with the azimuthal velocity of the outer cylinder $\left(\Omega_{o} \times r_{o}\right)$. The dashed lines connect the measured data points and the theoretical values at the walls

Coles and Van Atta (1966) performed laminar flow measurements with hot-wire anemometry, when only the outer cylinder is rotating at a constant speed with Reynolds numbers between $R e_{o}=2,000$ and 12,000. They reported a strong disturbance of the laminar flow in the mid-plane of axial direction, which is increasing with the Reynolds number. This distortion effect was not observed during our experiments, which is possibly due to the relatively low Reynolds number in our experiments. Ravelet et al. (2010) found that the error level between analytical calculations and measurements was higher close to the outer cylinder $\left(0.7 d \leq r-r_{i} \leq d\right)$. They concluded that the reason behind this is the refraction close to curved wall. However, for the results presented here, the disagreement is found to be of the same order for both regions close to the inner and 


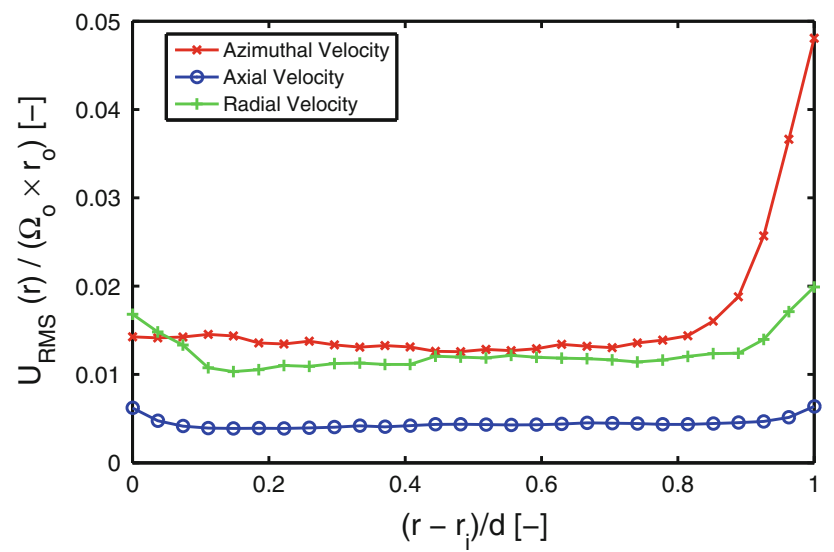

Fig. 6 RMS of velocities, based on the time-average of 150 instantaneous vector fields of laminar flow with only the outer cylinder rotating $\left(\Omega_{o}=0.48 \mathrm{rad} / \mathrm{sec}, \Omega_{i}=0, R e_{o}=643, R o=0.091\right.$, $\left.R e_{s}=615\right)$, as a function of the radial distance. All RMS values are normalised with the azimuthal velocity of the outer cylinder $\left(\Omega_{o} \times r_{o}\right)$

outer cylinder walls, with a slightly higher value near the inner cylinder. In our case, the camera viewing directions were much closer to the normal of the cylinder wall, which may have helped to eliminate the errors due to refraction.

In order to check the accuracy of the method, the RMS of the measured velocities were also calculated (Fig. 6). For the azimuthal velocity $v$, the RMS reaches its maximum value of $4.8 \%$ of the outer cylinder velocity. The maximum occurs at the outer cylinder wall. However, for $0 \leq r-r_{i} \leq 0.85 d$, it is always below $1.5 \%$. On the other hand, RMS values of axial and radial velocities are below 0.64 and $2.0 \%$, respectively, of the outer cylinder velocity. High RMS values for $0.85 d \leq r-r_{i} \leq d$ might be caused by the low $S N R$ close to walls. This is probably due to the effect of the boundaries of the measurement volume and ghost particles, which is explained in detail below. High RMS values might also be related with slight unroundness of the cylinder.

Particle image velocimetry measurements in the vicinity of the edges of the measurement domain are generally problematic. One of the reasons is the lower probability to find sufficient particle images in the interrogation window. A lower number of particles reduces the height of the correlation peak, which defines the measurement quality. This applies in particular to tomographic PIV, where the reconstruction is done in a volume that is slightly larger than the illuminated volume. Ghost particles are formed randomly throughout the reconstructed volume, whereas actual particles only exist in the illuminated volume. Although the number of the ghost particles remains constant, the ratio of the ghost particles to the actual particles in the interrogation windows becomes larger in the vicinity of the cylinder walls, where the interrogation windows

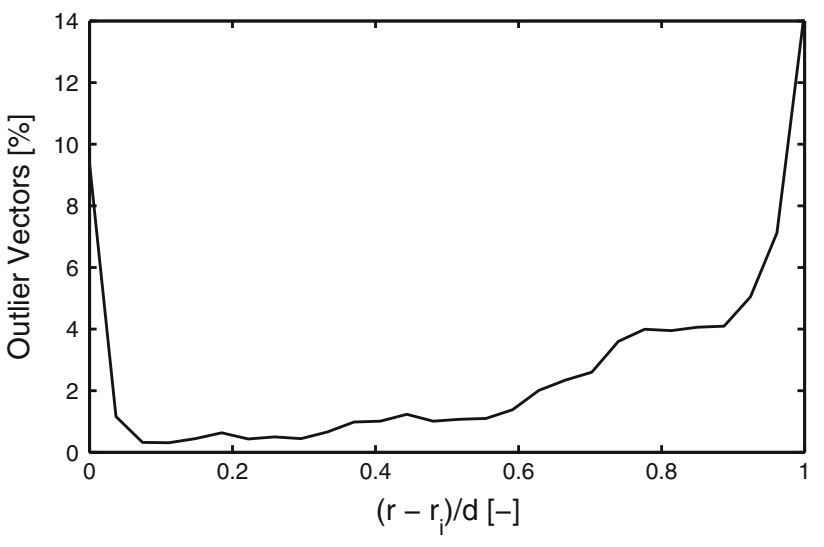

Fig. 7 An instantaneous example of percentage of outlier vectors to the all vectors in the same radial-axial cross-section, as a function of the radial distance. Calculated by $40 \times 40 \times 40$ voxels final interrogation window, with a $75 \%$ window overlap

partially overlap with the walls. The measured velocity component is affected by the presence of the ghost particles. Consequently, the signal strength, that is, the height of the correlation peak, is reduced in the vicinity of the inner and outer cylinder walls. This explains the higher error levels and the increase of the number of outliers near the cylinder walls. The Taylor-Couette setup has one more disadvantage. The tomographic reconstruction implementation that we use allows a reconstruction in a rectangular geometry only. In order to reconstruct the full measurement depth at positions where $\theta \neq 0$, one should include the external part of the cylinders of the Taylor-Couette setup (represented by the grey regions in Fig. 2).

One criterion that defines the quality of PIV measurements is the number of the invalid vectors per velocity field. The percentage of the invalid vectors to the valid vectors for an instantaneous velocity field is given in Fig. 7. Except for the regions close to the cylinder walls $\left(0 \leq r-r_{i} \leq 0.04 d\right.$ and $\left.0.85 d \leq r-r_{i} \leq d\right)$ the number of invalid vectors is below $4.1 \%$ of the total vectors. The value increases from 5 to $14 \%$ in the region $0.85 d \leq r-$ $r_{i} \leq d$. Since the percentage of the outliers are below $4 \%$ for most of the measurement volume, one can use slightly smaller interrogation windows for vector calculations in order to achieve a higher spatial resolution.

\subsection{Taylor vortex flow}

The measurements for Taylor vortex flow were performed at angular velocities of the outer and inner cylinders of $\Omega_{o}=0.38 \mathrm{rad} / \mathrm{sec}$ and $\Omega_{i}=0.88 \mathrm{rad} / \mathrm{sec}$, respectively. Corresponding Reynolds and rotation numbers are $R e_{s}=565$ and $R o=-0.231$ (Table 1). The measured flow profile based on an average over 300 instantaneous velocity fields is given in Fig. 8. In addition to vorticity 


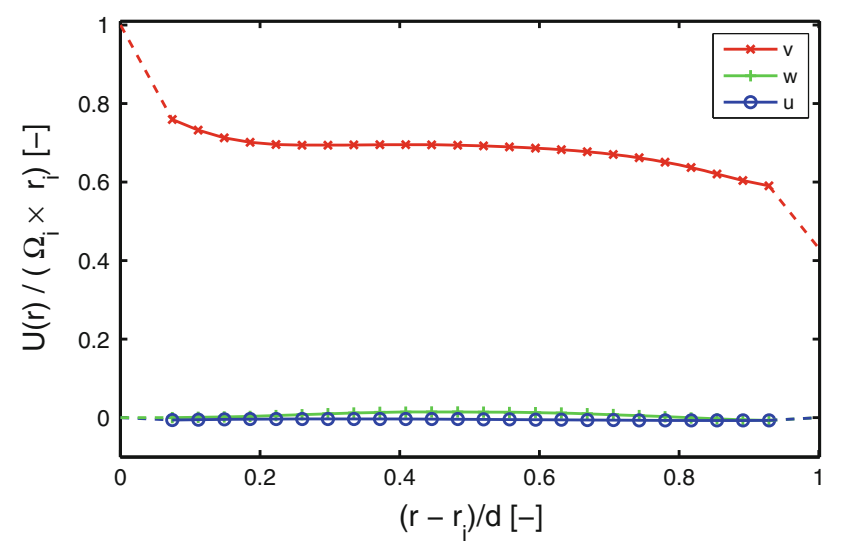

Fig. 8 Mean velocity of the Taylor vortex flow with co-rotation of cylinders $\quad\left(\Omega_{o}=0.38 \mathrm{rad} / \mathrm{sec}, \Omega_{i}=0.88 \mathrm{rad} / \mathrm{sec}, R e_{o}=500, R e_{i}=\right.$ $1,000, R o=-0.231, R e_{s}=565$ ), as a function of the radial position. Time-averaging was performed over 300 instantaneous vector fields. All velocities are normalised with the azimuthal velocity of the inner cylinder $\left(\Omega_{i} \times r_{i}\right)$. The dashed lines simply connect the measured data points to the theoretical values at the walls

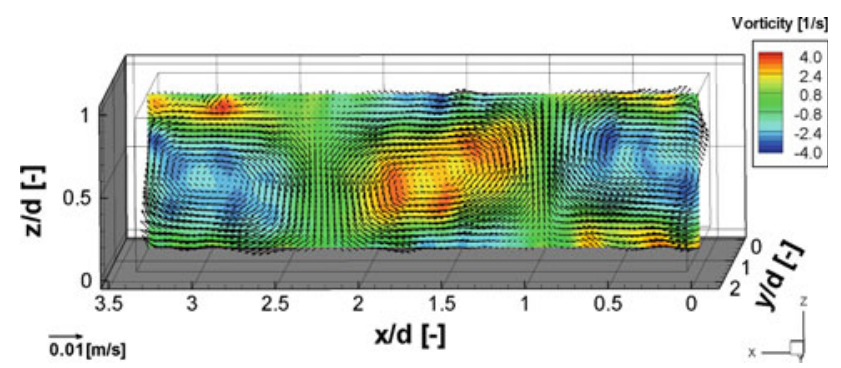

Fig. 9 Instantaneous representation of vorticity and velocity vectors for Taylor vortex flow $\left(R e_{o}=500, R e_{i}=1,000, R o=-0.231\right.$, $\left.R e_{s}=565\right)$, given at a cross-section at the center of the measurement volume in the azimuthal direction $(y)$. Vorticity in the azimuthal direction is color coded. Only the vectors that are tangential to the cross-sectional plane are given

calculations (Fig. 9), the vortical motion of the flow can be represented by means of the Q-criterion (Hunt et al. 1988), of which isosurfaces are shown in Fig. 10.
Two significant properties can be concluded from the plots. The first one is the inclined elliptical shape of the Taylor vortices. The inclination axes are making an angle of $\pm 25^{\circ}$ with the azimuthal direction of the cylinders. The high-velocity radial flow in between adjacent vortices might be responsible for the inclination of the vortex shapes. For instance, at $x / d \approx 1$ in Fig. 9, both positive and negative vorticity are tilted outwards. This can be associated with the strong outward flow in the radial direction between two vortices. Similarly, at $x / d \approx 2.3$ both positive and negative vorticity are inclined towards the inner cylinder, because of the strong radial inflow coming through this region. The strong inflow and outflow cause the tilting of the elliptical shape of the Taylor vortices. This is in agreement with the observations of Ravelet et al. (2010), where they reported a similar deformation for $R o \leq-0.04$. Smieszek and Egbers (2005) discussed similar, but lesssignificant deformation for $R e_{i}=259$ at $R o=-0.5$, but with a shorter cylinder height of $\Gamma=4.64$.

On the other hand, the inclined characteristics of the vortices resembles wavy vortex flow. Wang et al. (2005) reported inclination angles of $\pm 45^{\circ}$ for wavy vortex flow with a gap ratio of $\eta=0.733$. However, there is no evidence for a significant transfer of fluid between adjacent vortices in our measurements, which is a typical property of wavy vortex flow (Wereley and Lueptow 1998; Akonur and Lueptow 2003; Wang et al. 2005; Abcha et al. 2008). On the contrary, the boundaries of each individual coherent structure are well defined for the measurements presented here. Unlike wavy vortex flow (Wang et al. 2005), the boundaries between neighboring Taylor vortices are fairly stationary in our measurements.

The second property is the appearance of two concentrated regions with a high vorticity level inside each individual Taylor vortex structure. If we consider the Taylor vortex in the middle of the Q-plots in Fig. 10, the core of the vortical structure can be seen as divided into two vortices inside. This can be explained with the existence of two separate, highly concentrated, vortical regions inside
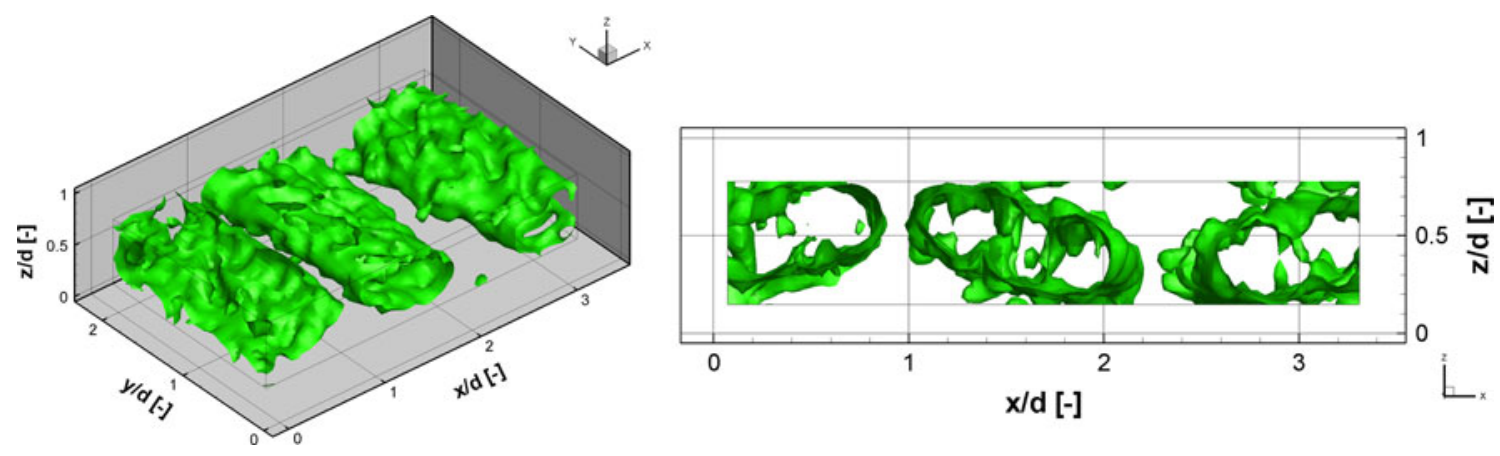

Fig. 10 The isosurfaces for constant values of the Q-criterion (Hunt et al. 1988) $\left(Q=0.25 \mathrm{~s}^{-2}\right)$ determined from the measured instantaneous flow fields of Taylor vortex flow $\left(R e_{o}=500, R e_{i}=1,000, R o=-0.231, R e_{s}=565\right)$. 3D view (left), side view in $x-z$ plane $(r i g h t)$ 

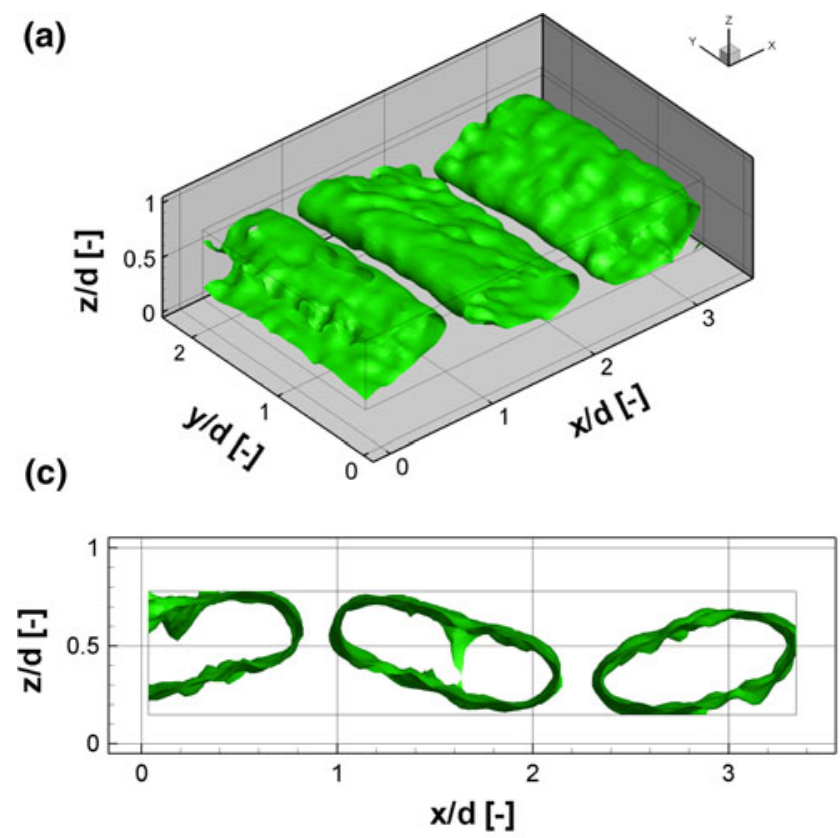

Fig. 11 Time-average of six successive instantaneous Taylor vortex flow fields. 3D view of isosurfaces for $Q$-criterion $\left(\mathrm{Q}=0.25 \mathrm{~s}^{-2}\right)(\mathbf{a})$, $x-y$ (axial-azimuthal) view $\left(\mathrm{Q}=1.5 \mathrm{~s}^{-2}\right)(\mathbf{b}), x-z$ view $(\mathrm{Q}=0.25$ $\left.\mathrm{s}^{-2}\right)(\mathbf{c})$, color coded vorticity values in azimuthal $y$ direction and

each individual Taylor vortex. The high-concentration regions become more obvious if we increase the value of Q-criterion isosurface for visualisation or perform timeaveraging. Any possible relation between the high concentration zones and the inclined shape of Taylor vortices will need to be confirmed in further studies.

Similar to Wereley and Lueptow (1998), we performed time averaging on six instantaneous Taylor vortex flow velocity vector fields (Fig. 11). The vortical structures thereby become smoother with respect to the plots for the instantaneous vector fields (Fig. 10). The inclined rectangular shape of the vortical structures is preserved in the averaging procedure. The two regions with a concentration of vorticity inside the individual Taylor vortices are also visible in the $x-y$ (axial-azimuthal) view, which is plotted for higher values of $Q$. The isosurface contours of the azimuthal velocity $(v)$ show sharper transitions than the ones represented by Wereley and Lueptow (1998). This is most likely due to the higher Reynolds numbers used in our measurements $\left(R e_{s}=103\right.$ and 124 vs. $R e_{s}=565$ for our data).

In addition to the observations above, the formation of new Taylor vortices was observed in our measurement as well. An example of the formation cycle is shown in Fig. 12. Initially, the leading edges of a pair of counterrotating vortical structures appear in the outflow region between two counter-rotating Taylor vortices. They are similar both in shape, size and vorticity strength. They (b)

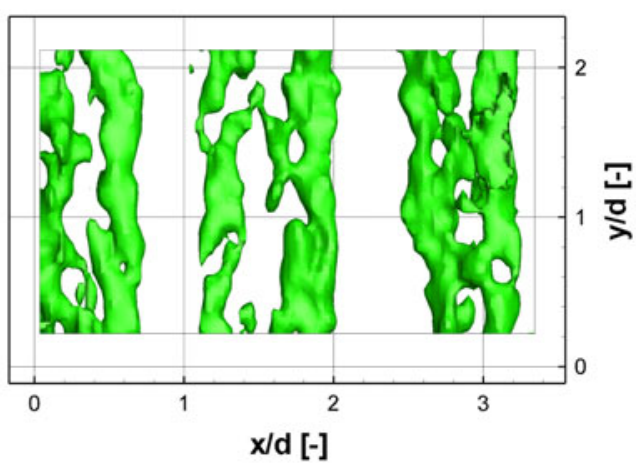

(d)

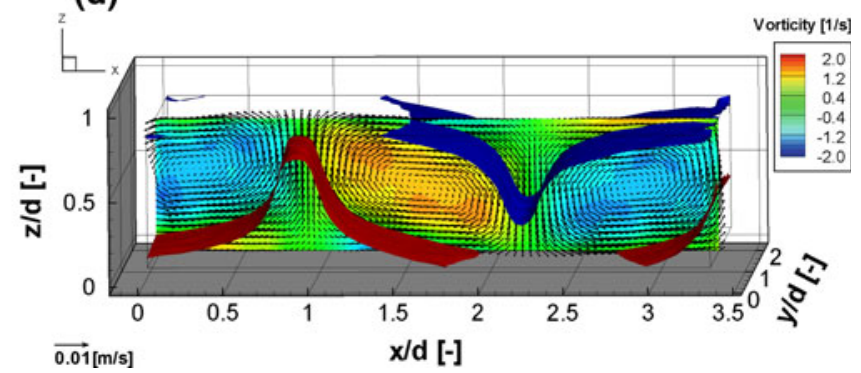

tangential velocity vectors in radial-axial plane plotted on the radialaxial cross-section alongside the azimuthal velocity isosurface contours (isosurface red: $-7.15 \times 10^{-2} \mathrm{~m} / \mathrm{s}$, isosurface blue: $\left.-6.25 \times 10^{-2} \mathrm{~m} / \mathrm{s}\right)(\mathbf{d})$

emerge in the region close to the inner cylinder wall and then move in the streamwise (azimuthal) flow direction. As they move forward, their size and diameter tend to expand, and they move to the center of the gap between cylinders. Their presence imposes the bigger vortices to move away from each other in axial direction. This progress continues until the diameter of the newly appeared vortical structures becomes equal to the diameter of the original structures. New counter-rotating vortical structures replace the previous ones at the end of the cycle. The opposite behaviour was observed as well. Disappearance of pairs of vortical structures follows the same cycle, but in reverse order.

In our measurements, the formation of new vortices always starts at the outflow region, while the disappearance always ends at the inflow region. Similarly, the leading edges of the new vortices appear in the vicinity of the inner cylinder, where the trailing edges of the disappearing vortices are close to the outer cylinder. It should be noted that these cycles were observed randomly both in time and space. However, it is not possible to make a guess of the appearance frequency. Thus, one should be careful when performing time-averaging over instantaneous Taylor vortex flow fields. The averaging can only be performed when the cores of the vortical structures remain at the same positions. Similar phenomena were reported by Coles (1965) as well. He briefly discussed single vortex filaments that first doubled themselves, then merged again into a single vortex filament. However, for our measurements, the 
(a)

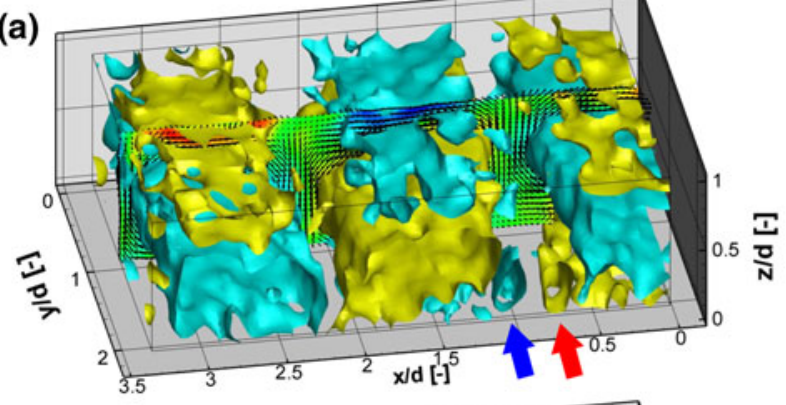

(c)
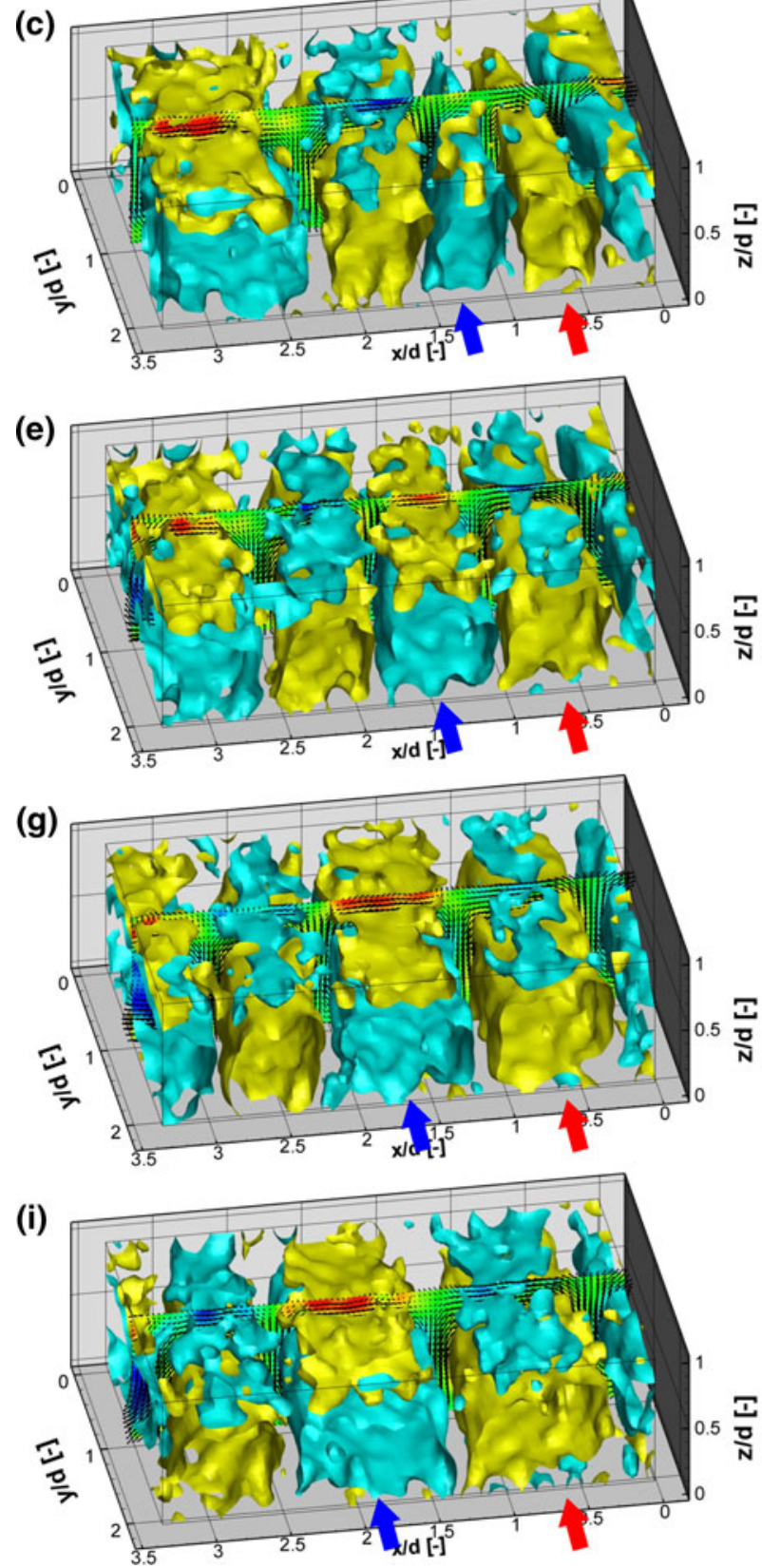

Fig. 12 3D representation of one cycle of the new vortex formation for Taylor vortex flow. Isosurfaces of constant vorticity values in the azimuthal direction $y$ (yellow: $0.75 \mathrm{~s}^{-1}$, blue: $-0.75 \mathrm{~s}^{-1}$ ). Blue and
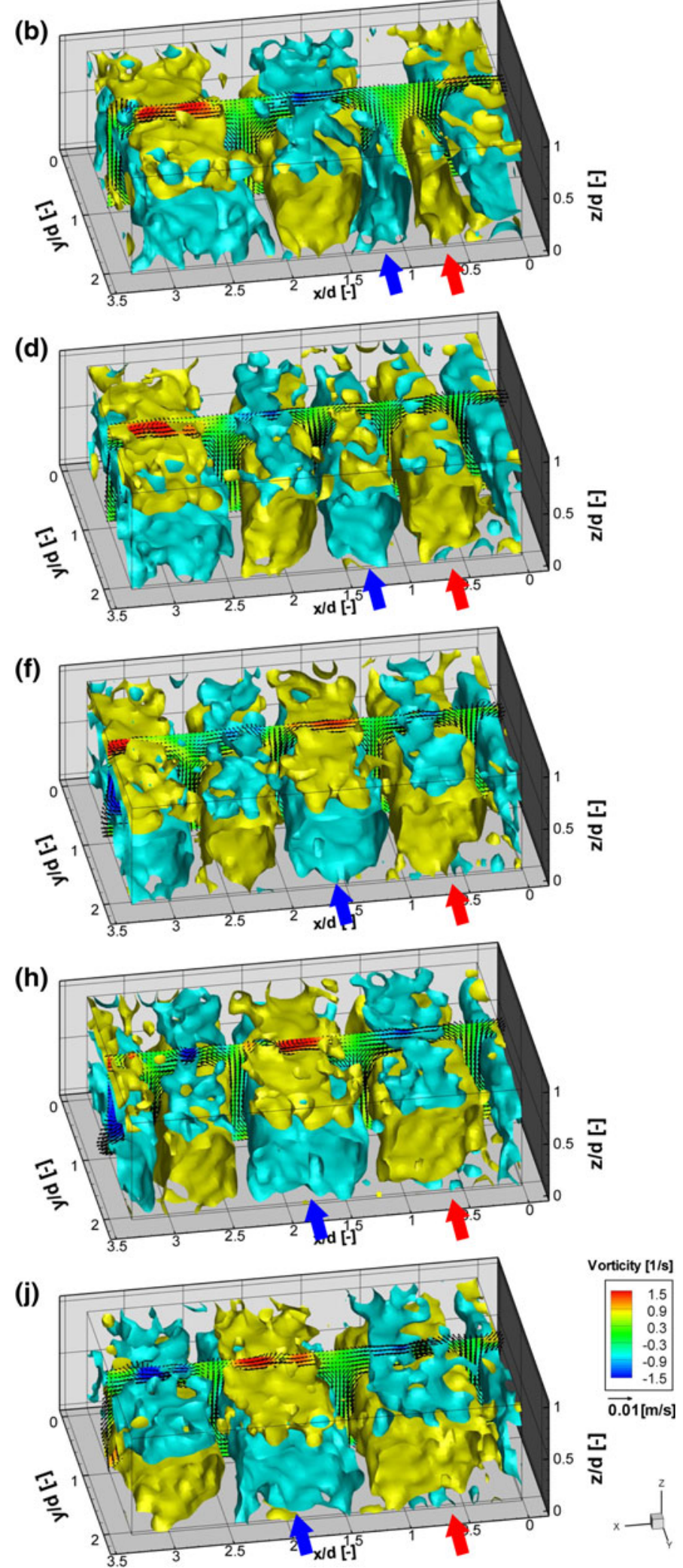

red arrows indicate the approximate centres of the new-forming vortical structures in the axial direction. The time differences between consequent images are $\Delta t=0.21 \mathrm{~s}$ 
phenomenon was not observed as a "doubling". It is more like an appearance or disappearance of new vortex pairs in between two counter-rotating vortices. Further investigation should be done to find out any possible relation between these two incidents. On the other hand, based on visualisation experiments in a Boger fluid, Smieszek and Egbers (2005) reported the continuous formation of new vortices in the middle of the axial position of the cylinders. They related the formation to the instability of the Taylor vortices. However, their observations on periodic movement of the vortex cores in the axial direction has not been observed in the measurements presented in this paper.

\subsection{Fully turbulent flow}

In this section, results for the characteristics of fully turbulent flow at a slightly positive $R o$ are discussed. In their paper, Andereck et al. (1986) defined fully turbulent flow as a region of turbulent flow without any apparent largescale structure and characterised the dominant length scale as smaller than the gap $d$ for high cylinder speeds. Since they could not identify obvious structures, they identified it as "featureless turbulence flow".

The measurements were performed when the outer and inner cylinders are rotating with angular velocities of $\Omega_{o}=-2.26 \mathrm{rad} / \mathrm{sec}$ and $\Omega_{i}=1.57 \mathrm{rad} / \mathrm{sec}$, respectively. The shear Reynolds number is $R e_{s}=4,700$. Corresponding Reynolds and rotation numbers, as well as the tomographic PIV measurement parameters are summarised in Table 1.

The measured velocity profile is given in Fig. 13. The characteristic of the azimuthal profile is similar to results reported in the literature (Vaezi et al. 1997; Dong 2008;

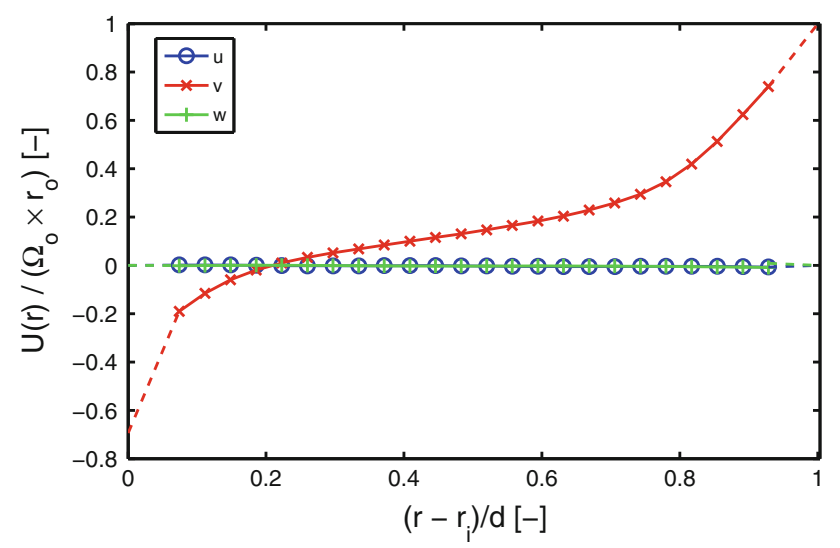

Fig. 13 Mean velocity profile of the fully turbulent flow with counterrotation of cylinders $\left(R e_{o}=-2,900, R e_{i}=1,850, R o=0.019\right.$, $\left.R e_{s}=4,700\right)$, as a function of the radial position. Time-averaging was performed over 150 instantaneous vector fields. Spatial averaging was performed in the axial direction of the cylinders. All velocities are normalised with the azimuthal velocity of the outer cylinder $\left(\Omega_{o} \times r_{o}\right)$. The dashed lines simply connect the measured data points and the theoretical values at the walls
Ravelet et al. 2010). Since $R o>0$, the azimuthal flow profile is not symmetric, and the plateau in the middle section is shifted in positive direction towards the velocity of the outer cylinder. The velocity near the outer cylinder wall is found to be underestimated by $11 \%$ and near the inner wall by $47 \%$ compared to their theoretical values $\left(\Omega_{o} \times r_{o}=-0.27 \mathrm{~m} / \mathrm{s}\right.$ for the outer and $\Omega_{i} \times r_{i}=0.17 \mathrm{~m} / \mathrm{s}$ for the inner cylinder walls). This is because of the thin near-wall layer that is not resolved and gradients are underestimated due to low resolution.

We measured a total of 300 vector fields to make a further analysis of the characteristics of the time-averaged velocity field for fully turbulent flow. In contrast to findings by Dong (2008), the time-averaged vector fields do not contain any apparent large structures like Taylor vortices. Dong (2008) explains the Taylor vortex-like structures in time-averaged field as the cumulative effect of instantaneous small-scale vortex organisation, which results in average structures similar to Taylor vortices.

If the instantaneous vector fields are considered, obvious structures similar to Taylor vortices were not observed in our measurements (Fig. 14). However, the flow fields contain disorganised small-scale and large-scale structures, as typical for a regular turbulent shear flow.

\section{Dissipation rate estimations}

In this section, we focus on the dissipation rate, which is computed from the velocity gradients estimated by tomographic PIV measurements. The validation is done using the analytically well-defined laminar flow. Moreover, the average dissipation rate is compared to the values that are obtained from torque measurements for increasing $R e_{s}$ numbers for fully turbulent flows. We discuss the actual spatial resolution of tomographic PIV based on the dissipation rate estimations.

The dissipation rate in Cartesian coordinates, which simplifies the calculations over cylindrical coordinates in our experiments (Lewis and Swinney 1999; Sharp et al. 2000; Sharp and Adrian 2001), is given by:

$$
\begin{aligned}
\varepsilon= & v\left\{2\left(\left\langle\left(\frac{\partial u}{\partial x}\right)^{2}\right\rangle+\left\langle\left(\frac{\partial v}{\partial y}\right)^{2}\right\rangle+\left\langle\left(\frac{\partial w}{\partial z}\right)^{2}\right\rangle\right)\right. \\
& +\left\langle\left(\frac{\partial u}{\partial y}\right)^{2}\right\rangle+\left\langle\left(\frac{\partial v}{\partial x}\right)^{2}\right\rangle+\left\langle\left(\frac{\partial u}{\partial z}\right)^{2}\right\rangle \\
& +\left\langle\left(\frac{\partial w}{\partial x}\right)^{2}\right\rangle+\left\langle\left(\frac{\partial v}{\partial z}\right)^{2}\right\rangle+\left\langle\left(\frac{\partial w}{\partial y}\right)^{2}\right\rangle \\
& \left.+2\left(\left\langle\frac{\partial u \partial v}{\partial y} \frac{\partial v}{\partial x}\right\rangle+\left\langle\frac{\partial u \partial w}{\partial z} \frac{\partial x}{\partial x}\right\rangle+\left\langle\frac{\partial v}{\partial z} \frac{\partial w}{\partial y}\right\rangle\right)\right\}
\end{aligned}
$$



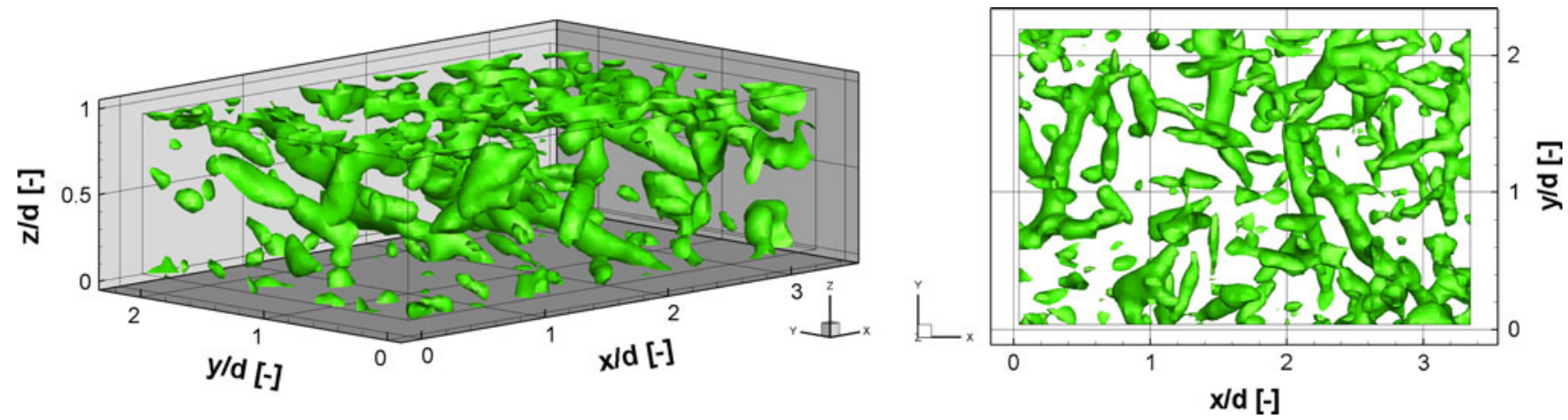

Fig. 14 The isosurfaces for constant values of Q-criterion (Hunt et al. 1988) $\left(\mathrm{Q}=400 \mathrm{~s}^{-2}\right)$ determined from the measured instantaneous flow fields of fully turbulent flow $\left(R e_{o}=-2,900\right.$,

where, " $\langle\bullet\rangle$ " represents a temporal ensemble average. The $u, v$ and $w$ values are the instantaneous velocities in the $x, y$ and $z$ directions, respectively. McEligot et al. (2008) showed that in case of the dissipation rate estimations, the fluctuating velocity components are dominant in the bulk flow, while the mean flow is important in the regions close to the wall. However, since we aimed to compute the total dissipation, the instantaneous velocities, which include both the mean and the fluctuating velocity components, are used for the estimations.

Different to the previous efforts to compute the dissipation rate using 2D PIV measurements (Sheng et al. 2000; Sharp et al. 2000; Sharp and Adrian 2001; Baldi and Yianneskis 2003; Racina and Kind 2006), all instantaneous $3 \mathrm{D}$ velocity gradients are known via tomographic PIV. As a consequence, assumptions based on flow axisymmetry or isotropy are not necessary, and only directly measured values are used to compute $\varepsilon$ (Baldi and Yianneskis 2003). The precision of the computations is mainly limited by the spatial resolution of the measurements.

The computed dissipation rates are compared against values for $\varepsilon$ based on separate torque measurements (Racina and Kind 2006). For Taylor-Couette flow, the total dissipation of kinetic energy per unit time must be equal to the power supplied by the rotating cylinders. Furthermore, the balance of momentum in the steady state requires the torque magnitude on the inner and outer cylinder to be equal, which reduces the expression for the mean dissipation rate per unit volume to:

$\overline{\varepsilon_{T}}=\frac{P}{\rho V}=\frac{T\left|\Omega_{i}-\Omega_{o}\right|}{\rho V}$

where $P$ stands for the power input due to the inner cylinder rotation, $T$ represents the torque measured on the inner cylinder, $\Omega_{i}$ and $\Omega_{o}$ are the angular velocities of inner and outer cylinders, $\rho$ is the density of the fluid and $V$ is the total volume of the fluid in the Taylor-Couette setup.
$R e_{i}=1,850, R o=0.019, R_{s}=4,700$ ); 3D view (left), side view in $x-y$ (axial-azimuthal) plane (right)

In order to compute the velocity gradients in (6), a second-order polynomial regression to the measured velocity field was done (Elsinga et al. 2010):

$$
\begin{aligned}
f_{\text {reg }}\left(r_{x}, r_{y}, r_{z}\right)= & a_{0}+a_{1} r_{x}+a_{2} r_{y}+a_{3} r_{z}+a_{4} r_{x} r_{y}+a_{5} r_{x} r_{z} \\
& +a_{6} r_{y} r_{z}+a_{7} r_{x}^{2}+a_{8} r_{y}^{2}+a_{9} r_{z}^{2}
\end{aligned}
$$

where $r_{x}, r_{y}$ and $r_{z}$ are the relative distances from a point in the $x, y$ and $z$ directions, respectively. The method fits a second-order polynomial function to the velocity distribution in a $5 \times 5 \times 5$ neighbourhood around a point $\left(x_{1}, y_{1}, z_{1}\right)$, which acts like a filter as well (Elsinga et al. 2010). The fit parameters $a_{1}, a_{2}$ and $a_{3}$ represent the velocity gradients at $\left(x_{1}, y_{1}, z_{1}\right)$ in the $x, y, z$ directions, respectively.

The PIV method encounters problems to properly resolve Kolmogorov microscales, because of the limit of the spatial resolution (Sheng et al. 2000; Sharp and Adrian 2001; Baldi and Yianneskis 2003; Racina and Kind 2006; Tanaka and Eaton 2007; Lavoie et al. 2007; Adrian and Westerweel 2011). The Kolmogorov microscale is defined as:

$\lambda_{K} \equiv\left(\frac{\nu^{3}}{\overline{\varepsilon_{T}}}\right)^{1 / 4}$

where $v$ is the kinematic viscosity and $\overline{\varepsilon_{T}}$ is the mean dissipation rate estimated from torque data.

Small-scale fluctuations are filtered out when the space between the vectors $\left(\delta_{x}\right)$ is larger than the Kolmogorov length scale (Sheng et al. 2000; Tanaka and Eaton 2007). This results in the underestimation of turbulent kinetic energy dissipation rate (Sheng et al. 2000; Racina and Kind 2006; Tanaka and Eaton 2007). In contrast, for cases where $\delta_{x}$ is smaller than the Kolmogorov scale length, a decreasing $\delta_{x}$ leads to a rapid increase of dissipation rate because of the measurement noise. This noise is due to the finite measurement error (Saarenrinne and Piirto 2000; Tanaka and Eaton 2007). 
A measure of the smallest turbulent length scale that is captured by tomographic PIV $(\lambda)$ can be estimated by using (9). However, $\overline{\varepsilon_{T}}$ should be replaced by the mean dissipation rate computed by tomographic PIV (i.e. $\bar{\varepsilon}$ ). The effect of the spatial resolution to the dissipation rate estimations can be evaluated by $\lambda / \lambda_{K}$ ratio. The spatial resolution of the measurement is better if the ratio is closer to unity. Nevertheless, the ratio of $\lambda / \lambda_{K}$ is indicative of how well the flow has been resolved with respect to the dissipation rate estimation.

\subsection{Laminar flow and assessment of dissipation rate estimations}

Local dissipation rate estimations for the laminar flow are given in Fig. 15. They were computed with two different approaches. The first approach is to compute the gradients and the dissipation rates from a single, time-averaged vector field, which we refer to as "method 1". Since the time-averaging smooths the vector field, this method results in slightly lower values of velocity gradients and local dissipation rates.

The second approach, that is indicated as "method 2", is to compute the gradients and the dissipation rates for each of the 150 instantaneous vector fields individually, and then average the dissipation rates. In general, "method 2" results in slightly higher dissipation rates, since it includes the contribution of random noise in the measured velocity. The difference between these two methods is also plotted in Fig. 15. Both methods yield data that are in good agreement in the inner region. The difference is below $3.4 \%$ of the maximum dissipation rate in the region $0.1 d \leq r-r_{i} \leq 0.85 d$, which is associated to PIV noise. It is obvious that the difference between the results of the two

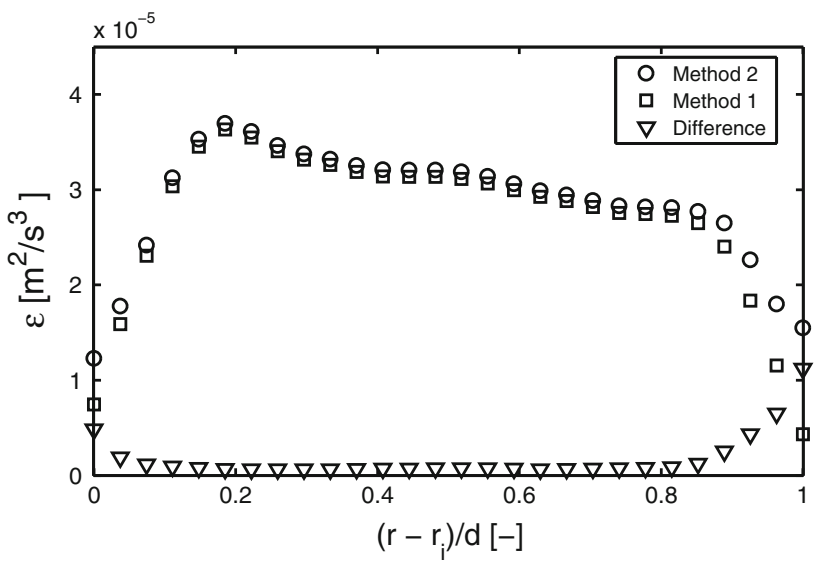

Fig. 15 Local dissipation rate estimations for laminar flow, obtained by tomographic PIV, as a function of radial position. Calculations were performed with two methods. Results were plotted along centerline in radial direction methods is higher close to the cylinder walls. The difference between the methods remains small however, indicating that the effect of random measurement noise is not significant. Since the other flow regimes are unsteady, only "method 2 " is considered in the remainder of this paper.

The analysis of individual velocity gradients reveals two dominant gradients, which are $\partial v / \partial z$ and $\partial w / \partial y$. These gradients are an order of 10 times higher than the remaining ones. However, all of the gradients were included in dissipation rate estimations. The "wavy" characteristics of the local dissipation rates is caused by the steplike behaviour of the gradient $\partial w / \partial y$, which might be due to peak locking effect (Adrian and Westerweel 2011). Postprocessing methods to correct the peak locking effect can be found in the literature (Roth and Katz 2001; Cholemari 2007). Since we aim to use the raw data without any correction, these methods were not implemented in this paper.

Obviously, the measured local dissipation rates in Fig. 15 significantly decrease towards the walls. This is due to the error caused during the velocity gradient estimation. A second-order polynomial regression uses a $5 \times 5 \times 5$ neighbourhood of vectors at each point. Therefore, the effect of the gradients at the borders of the domain (i.e. the cylinder walls) at both sides expands towards the inner section of the gap. This continues until the edges no longer are part of the domain of the $5 \times 5 \times 5$ kernel, which is the fourth data point from cylinder walls in the radial direction for our case. This effect was tested by excluding two and three data points from measurement domain at both sides for the velocity gradient estimations and is plotted in Fig. 16. The local dissipation rate in the region $0.18 d \leq r-r_{i} \leq 0.81 d$ was identical for both estimations. However, the deviation between two domains is large close towards each wall. In the case of excluding of two data points from each side, the effect of the cylinder walls is still dominant in the domain. Thus, similar to Worth et al. (2010), we decided to continue with excluding three data points for the analyses with $75 \%$ overlap, unless otherwise stated (see Sect. 5.2 and Table 4 for the exceptions). It should be noted that for all of the mean dissipation rate estimations presented in this paper, we used the local dissipation rates at the central plane of the measurement volume in the azimuthal direction (i.e. $\theta=0$ ). Additionally, we performed spatial averaging in the axial direction in the measurement volume, which is a homogeneous direction in our system.

As the laminar flow has a well-defined analytical form, local dissipation rate estimations based on the measurement of tomographic PIV can be compared with the analytical calculations of the local dissipation rate. The grid points of the velocity vectors obtained by tomographic PIV were used for analytical computations. We generated a volumetric domain of the analytical solution, which has the 


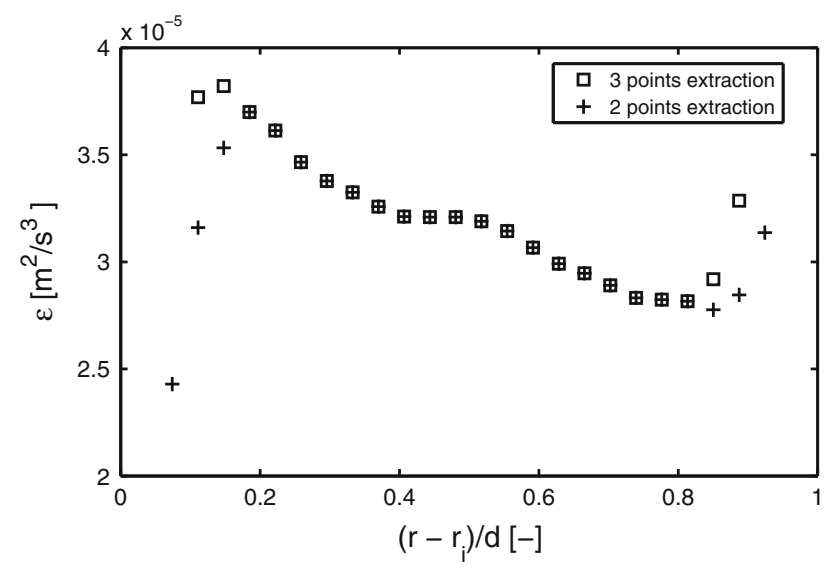

Fig. 16 The effect of borders to the local dissipation rate estimations for laminar flow, as a function of radial position

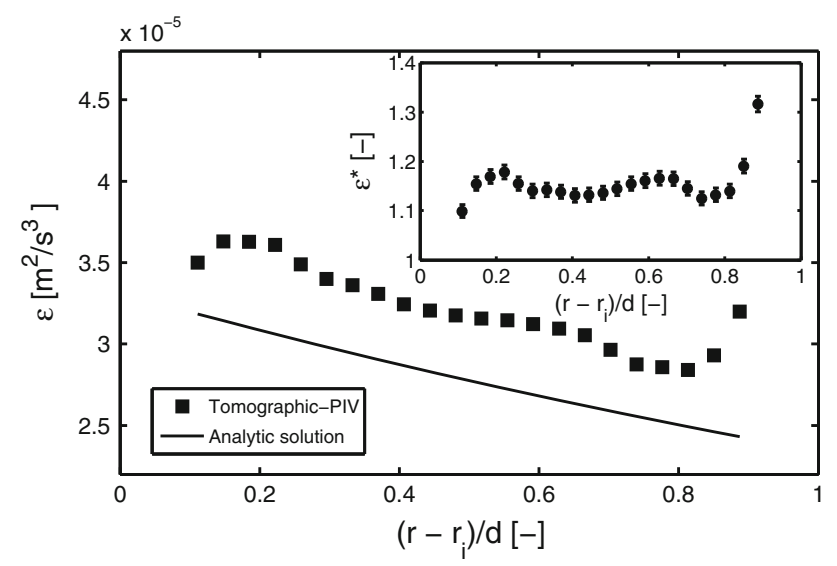

Fig. 17 Dissipation rate estimations, computed analytically and with tomographic PIV data, for laminar flow. Plotted as a function of radial position. Spatial averaging was performed in the axial direction of the cylinders. (Inset) Normalised dissipation rate estimations for laminar flow as a function of radial position. Normalisation was performed according to the analytically computed local dissipation rates. Error bars are representing the effect of uncertainty of the kinematic viscosity due to $\pm 0.5^{\circ} \mathrm{C}$ temperature difference

exact grid positions with the measured domain. Then, the same procedure for the dissipation rate estimation by tomographic PIV was followed on the analytically generated velocity vector domain. The results are shown in Fig. 17. The local dissipation rates estimated by tomographic PIV measurements are in good agreement with analytical results. The decreasing trend through the gap is consistent as well.

In order to make a quantitative comparison, the values estimated from the tomographic PIV data were normalised with the analytical result. The normalisation of the local dissipation rate with the analytical value is given as:

$\varepsilon^{*}=\frac{\varepsilon}{\varepsilon_{A}}$ where $\varepsilon$ represents the local dissipation rate estimated by tomographic PIV and $\varepsilon_{A}$ stands for the local dissipation rate calculated analytically. The plot for the normalised local dissipation rate is given in the inset of Fig. 17. The normalisation points out that the tomographic PIV measurements overestimates the local dissipation rate values everywhere. However, the difference is below $20 \%$ of the local dissipation rate, with the exception of a few data points near the cylinder walls. On the other hand, the effect of the uncertainty of the water temperature to the dissipation rate estimations are represented by error bars in the inset of Fig. 17. The uncertainty caused by the fluctuation of the fluid temperature is relatively small compared to the deviance from the analytical solution. Hence, we can conclude that the error in the dissipation rate estimations of the laminar flow case is mostly due to the errors in the measurements.

Even though the comparison with the analytical solution is the simplest and most reliable method for the validation of the dissipation rate estimation, it is not feasible for all flow types. Another approach should be used especially for the fully turbulent flows.

An advantage of the Taylor-Couette setup is the possibility of performing torque measurements. For the experimental setup presented here, the torque of the inner cylinder can be measured by a torque-meter that co-rotates with the inner cylinder shaft. More detailed information about the torque measurements on the current experimental setup is given by Ravelet et al. (2010). Using (7), a direct comparison between the mean dissipation rate obtained by tomographic PIV and by the torque measurement can be performed. It should be noted that the working fluid in our experiments was water, and the system was operated at relatively low rotating frequencies. As a consequence, the torque values are much lower than the limits of the measurement capability of the torque-meter. Therefore, a torque scaling of the data presented by Delfos et al. (2009) and Ravelet et al. (2010) was used instead. If (7) and (10) are combined, we obtain the normalisation of mean dissipation rate:

$\overline{\varepsilon^{*}}=\frac{\bar{\varepsilon}}{\overline{\varepsilon_{T}}}$

Another question arises in the computations of the mean dissipation rate, $\bar{\varepsilon}$. Because of the reasons explained above, the dissipation rates close to the cylinder walls cannot be estimated via tomographic PIV. However, these values are needed for computing the mean dissipation for the complete system. In order to estimate these values, a linear polynomial curve fitting operation was performed on the dissipation rate values on the radial direction. By the help of these polynomials, we estimated the dissipation 
Table 2 Results of the dissipation rate estimations for laminar flow $\left(R e_{s}=615\right)$, Taylor vortex flow $\left(R e_{s}=565\right)$, and fully turbulent flows

\begin{tabular}{llllllllllll}
\hline$R e_{S}$ & $\overline{\varepsilon_{A}}\left(\mathrm{~m}^{2} / \mathrm{s}^{3}\right)$ & $\overline{\varepsilon_{T}}\left(\mathrm{~m}^{2} / \mathrm{s}^{3}\right)$ & $\bar{\varepsilon}\left(\mathrm{m}^{2} / \mathrm{s}^{3}\right)$ & $\overline{\varepsilon^{*}}(-)$ & $\delta_{x}(\mathrm{~mm})$ & $\lambda_{K}(\mathrm{~mm})$ & $\delta_{x} / \lambda_{K}(-)$ & $\lambda(\mathrm{mm})$ & $\lambda / \lambda_{K}(-)$ & $\overline{\varepsilon_{S G S}}\left(\mathrm{~m}^{2} / \mathrm{s}^{3}\right)$ & $\overline{\varepsilon_{S G S}^{*}}(-)$ \\
\hline 615 & $2.79 \times 10^{-5}$ & $2.76 \times 10^{-5}$ & $3.19 \times 10^{-5}$ & 1.157 & 0.370 & {$[0.404]$} & {$[0.92]$} & {$[0.390]$} & {$[0.96]$} & - \\
565 & - & $2.85 \times 10^{-5}$ & $2.46 \times 10^{-5}$ & 0.862 & 0.370 & 0.412 & 0.90 & 0.427 & 1.04 & - \\
3,800 & - & $3.70 \times 10^{-3}$ & $1.97 \times 10^{-3}$ & 0.532 & 0.391 & 0.124 & 3.15 & 0.145 & 1.17 & $6.03 \times 10^{-3}$ & 1.629 \\
6,200 & - & $1.42 \times 10^{-2}$ & $3.05 \times 10^{-3}$ & 0.214 & 0.391 & 0.089 & 4.41 & 0.130 & 1.47 & $9.80 \times 10^{-3}$ & 0.690 \\
11,000 & - & $6.82 \times 10^{-2}$ & $7.25 \times 10^{-3}$ & 0.106 & 0.391 & 0.060 & 6.53 & 0.105 & 1.75 & $3.42 \times 10^{-2}$ & 0.502 \\
14,000 & - & $1.33 \times 10^{-1}$ & $1.12 \times 10^{-2}$ & 0.084 & 0.391 & 0.051 & 7.72 & 0.094 & 1.86 & $6.64 \times 10^{-2}$ & 0.498 \\
17,000 & - & $2.27 \times 10^{-1}$ & $1.58 \times 10^{-2}$ & 0.070 & 0.391 & 0.044 & 8.83 & 0.086 & 1.95 & $1.12 \times 10^{-1}$ & 0.491 \\
29,000 & - & $9.65 \times 10^{-1}$ & $3.88 \times 10^{-2}$ & 0.040 & 0.391 & 0.031 & 12.67 & 0.069 & 2.23 & $4.20 \times 10^{-1}$ & 0.435 \\
36,000 & - & 1.78 & $5.98 \times 10^{-2}$ & 0.034 & 0.391 & 0.027 & 14.76 & 0.062 & 2.34 & $9.50 \times 10^{-1}$ & 0.534 \\
47,000 & - & 3.75 & $1.19 \times 10^{-1}$ & 0.032 & 0.391 & 0.022 & 17.78 & 0.051 & 2.37 & 3.09 \\
\hline
\end{tabular}

$\overline{\varepsilon_{A}}$ is the analytical estimation of mean dissipation in a unit volume, $\overline{\varepsilon_{T}}$ is the mean dissipation estimation from torque data, $\bar{\varepsilon}$ is the mean dissipation estimation from tomographic PIV data. $\overline{\varepsilon_{S G S}}$ is the mean dissipation estimation from tomographic PIV data, using large eddy method. $\overline{\varepsilon^{*}}$ and $\overline{\varepsilon_{S G S}^{*}}$ are the normalised values of $\bar{\varepsilon}$ and $\overline{\varepsilon_{S G S}}$, respectively. $\delta_{x}$ represents the distance between the vectors, $\lambda_{K}$ is the Kolmogorov length scale computed from torque data, $\lambda$ is the equivalent Kolmogorov length scale estimated from the tomographic PIV data. We used $40 \times 40 \times 40$ voxel $^{3}$ final interrogation windows with $75 \%$ overlap. Hence, the linear dimension of the interrogation window, $D_{I}$, is 4 times the vector spacing (i.e. $D_{I}=4 \delta_{x}$ ). It is emphasised that the numerical values inside "[ $\bullet$ ]" do not represent an actual turbulence characteristic, but are evaluated for the aim of comparison. Similarly, since the large eddy method is only valid for turbulent flows, the computations with the large eddy method are excluded for the laminar and Taylor vortex flow

rates at the cylinder walls. For the laminar flow case, we used all data points of the domain to fit the equation. Since the analytical solution shows approximately linear behaviour, this approach seems reasonable. However, as it will be discussed in the next section, the dissipation rates of the fully turbulent flows have different characteristics to that of the laminar flow. Thus, using the complete domain for the curve fitting would result in erroneous estimations for the turbulent flow cases. Hence, we used 3 data points, which are the closest to the cylinder walls, to fit linear polynomials. Two separate polynomials, one for the inner cylinder and another one for the outer cylinder, were used for each case to estimate the dissipation rates at the inner and outer cylinder walls. In order to compute the mean dissipation of the Taylor-Couette system, numerical integration was performed over the measured data points in the middle region and the estimated values on the walls.

A summary of the dissipation rate estimations for all flow cases are presented in Table 2. Note that, for the laminar case, the Kolmogorov length scale-related values in Table 2 (i.e. $\lambda_{K}, \delta_{x} / \lambda_{K}, \lambda$ and $\lambda / \lambda_{K}$ ), are meaningless in terms of "turbulence" characteristics. However, they are presented for the aim of comparison. The mean dissipation rate for the laminar flow case $\left(R e_{s}=615\right)$ is in good agreement with both estimations of the analytical value and the scaled torque data. The error level is of the order of $15 \%$. Since the equivalent "Kolmogorov length scale" is relatively large for the laminar case, the vector spacing is smaller than this $\left(\delta_{x} / \lambda_{K}=0.915\right)$. Thus, the overestimation is caused by the noise during the measurements (Saarenrinne and Piirto 2000; Racina and Kind 2006; Tanaka and
Eaton 2007; Adrian and Westerweel 2011; Buxton et al. 2011). Tanaka and Eaton (2007) reported an error level of 20-30\% at a similar $\delta_{x} / \lambda_{K}$, but with a correction. We can conclude that, without any need for a further correction, tomographic PIV has a similar order of error as for corrected 2D PIV estimations.

The different nature of the torque and tomographic PIV methods might lead to a some degree of uncertainty. Comparison of both measurements with the analytically calculated mean dissipation rate indicates an uncertainty of the torque measurements as $\approx 1 \%$ and the tomographic PIV measurements as $\approx 15 \%$.

The mean dissipation rate estimation for the Taylor vortex flow $\left(R e_{s}=565\right)$ is similar to the laminar flow case. As expected, measurements are very close to resolving the flow $\left(\lambda / \lambda_{K}=1.04\right)$. The error level is of the order of $15 \%$ again, but the dissipation rate is now slightly underestimated. Since both large-scale flow cases have similar error levels, the discrepancy might be due to the contribution of the errors in the tomographic PIV and other effects in the estimations.

\subsection{Fully turbulent flows}

In this section, the fully turbulent flows are evaluated, where we aim to investigate the relation between turbulent length scales and the spatial resolution of the tomographic PIV measurements, using three different methods. Initially, in order to understand the effect of the turbulence intensity to the dissipation rate estimations, the size of the interrogation window ( $I W$ in voxel $-D_{I}$ in $\mathrm{mm}$ ) was kept 
constant. In the mean time, $R e_{s}$ was increased from 3,800 to 47,000 , at an exact counter rotation of the cylinders; $R o=0$ (Sect. 5.2.1). This approach results in measurements with constant spatial resolution, but decreasing Kolmogorov length scales.

In the second approach (Sect. 5.2.2), the size of the interrogation window was increased, while the shear Reynolds number remained constant. In this way, $\lambda_{K}$ was kept constant, while $\delta_{x}$ increased. Worth et al. (2010) performed a similar analysis, but using DNS data at a single Reynolds number.

Finally, in Sect. 5.2.3, the shear Reynolds number is kept constant, and we discuss the influence of different interrogation window overlap values while maintaining constant interrogation window size. This has the same effect on $\delta_{x}$ and $\lambda_{K}$ as it has in the second approach. However, this approach allows to evaluate the effect of oversampling to the measurement result. Additionally, in Sect. 5.2.4, we compare the dissipation rate estimates with the estimates computed by the large eddy PIV method (Sheng et al. 2000; Sharp and Adrian 2001).

Estimations of dissipation rate for fully turbulent flows were performed over 1,000 instantaneous velocity fields, which is sufficiently higher than the required number of samples for statistically reliable results (Baldi and Yianneskis 2003). Our tests (Fig. 18) show that an uncertainty below $4 \%$ requires at least 150 independent vector fields, where the uncertainty level becomes lower than $1 \%$ when at least 650 vector fields are used. From this, we conclude that the sampling error of 1,000 vector fields is below $1 \%$.

\subsubsection{Effect of Reynolds number}

The results of the dissipation rate estimations for increasing shear Reynolds numbers are plotted in Fig. 19. The plots show the characteristics of the local dissipation rates along the gap between the cylinders. The dissipation rates are

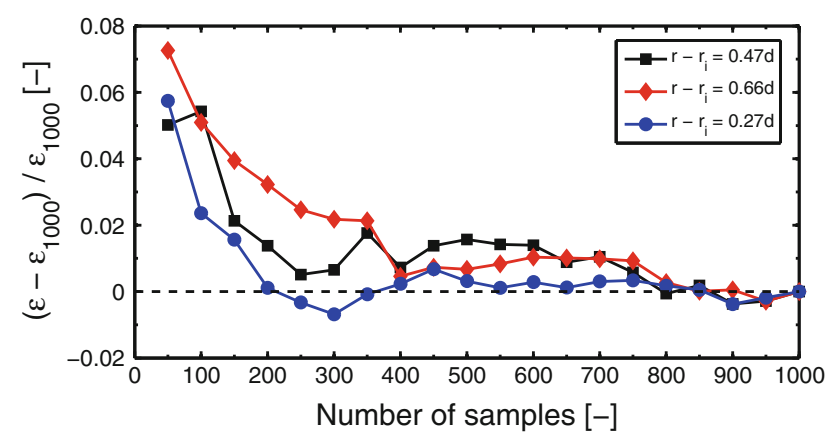

Fig. 18 Convergence of the average dissipation rate $(\varepsilon)$ with the number of frames compared to the average of 1,000 frames $\left(\varepsilon_{1000}\right)$, at three radial positions. Computations were performed at $R e_{s}=14,000$ with $40 \times 40 \times 40$ voxel $^{3}$ final interrogation window and $75 \%$ overlap

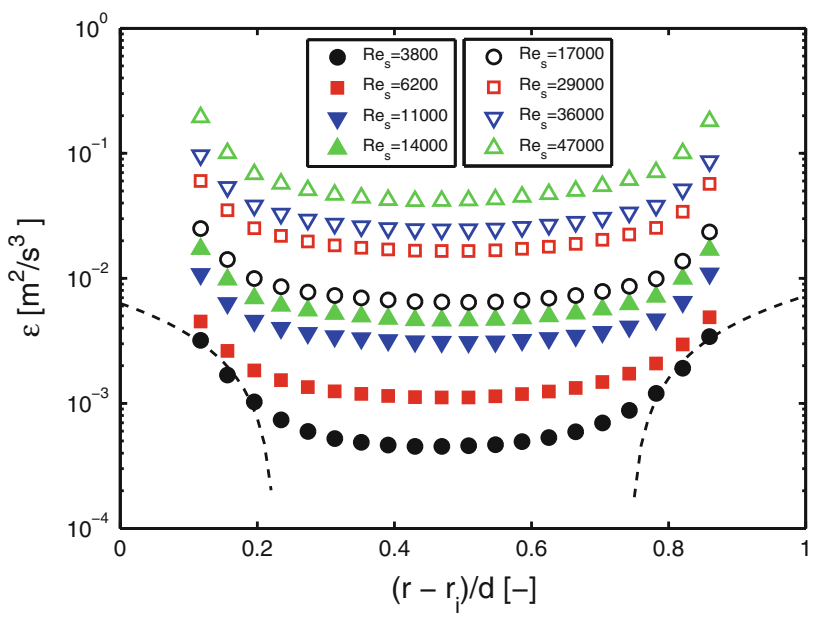

Fig. 19 Dissipation rate estimations for fully turbulent flow cases $\left(R e_{s}=3,800-47,000\right)$ with exact counter-rotation of the cylinders. Profiles were computed with $40 \times 40 \times 40$ voxel $^{3}$ final interrogation window and $75 \%$ overlap. Spatial averaging was performed in the axial direction of the cylinders. Three data points in each direction near the flow boundaries were excluded in order to avoid the effect of the boundaries of the domain on the results for the estimation of the dissipation rate. Dashed lines represent the linear extrapolation to estimate the wall value for $R e_{s}=3,800$

increasing for increasing $R e_{s}$, as expected. The ratio between the dissipation rates at $R e_{s}=3,800$ and 47,000 is of the order of $10^{2}$ for the estimations via tomographic PIV. Furthermore, for each shear Reynolds number, the local dissipation rates are almost symmetrical with respect to the $r-r_{i}=0.5 d$ plane. The regions close to the inner and the outer cylinder have similar rates of dissipation, and they are higher than for the middle section. The local dissipation rate values reveal a plateau at the middle center region. The difference between the minimum and the maximum dissipation rates for each profile decreases for increasing $R e_{s}$. Hence, the plateau becomes flatter for increasing values of $R e_{s}$.

As explained before, unlike the case for laminar flow, it is not possible to compute the dissipation rates analytically for fully turbulent flows. However, the mean dissipation was estimated using the torque data measured with the same experimental setup by Delfos et al. (2009) and Ravelet et al. (2010). Results for the estimations of the mean dissipation rate are given in Table 2 .

The agreement between the estimates for the mean dissipation rate via torque scaling and tomographic PIV measurements are not as good as for the laminar flow case. Tomographic PIV underestimates the mean dissipation by $47 \%$ for the best case $\left(R e_{s}=3,800\right)$, and up to $97 \%$ for higher values of $R e_{s}$. Mainly, this is the result of the finite spatial resolution of the tomographic PIV data. If the Kolmogorov scale is less than the vector spacing (i.e. $\delta_{x} / \lambda_{K}>1$ ), the dissipation rate values would be underestimated (Sheng 
et al. 2000; Tanaka and Eaton 2007). This is due to uncaptured small-scaled structures (Lavoie et al. 2007).

In the literature, various values of dissipation rate estimation errors have been reported. Tanaka and Eaton (2007) demonstrated that at high spatial resolutions, the error level might reach up to 10 times the actual dissipation value and for lower spatial resolutions the dissipation rates are underestimated. Sharp and Adrian (2001) estimated the contribution of the unresolved scales using a Smagorinsky model. They concluded that approximately $70 \%$ of the turbulent dissipation had been captured in their measurements at a spatial resolution of about 8 Kolmogorov length scales. Tomographic PIV returns similar errors at comparable spatial resolution. Racina and Kind (2006) showed lower mean dissipation rates obtained from 2D PIV data with decreasing resolution, but the results are somewhat difficult to compare due to the different nature of the flow, that is, wavy vortex flow compared to fully turbulent here.

\subsubsection{Effect of the size of the interrogation window}

In order to isolate the effect of the interrogation window size on the velocity vector, calculations are performed on the very same measurement data with different final interrogation window sizes. This way, the Kolmogorov length scale, $\lambda_{K}$, is maintained constant. We performed this analysis for $R e_{s}=3,800,14,000$ and 47,000.

The local dissipation rate estimations for $R e_{s}=14,000$ with different interrogation window sizes are plotted in Fig. 20. Since the characteristics are similar to those for $R e_{s}=3,800$ and 47,000, we omitted those in Fig. 20.

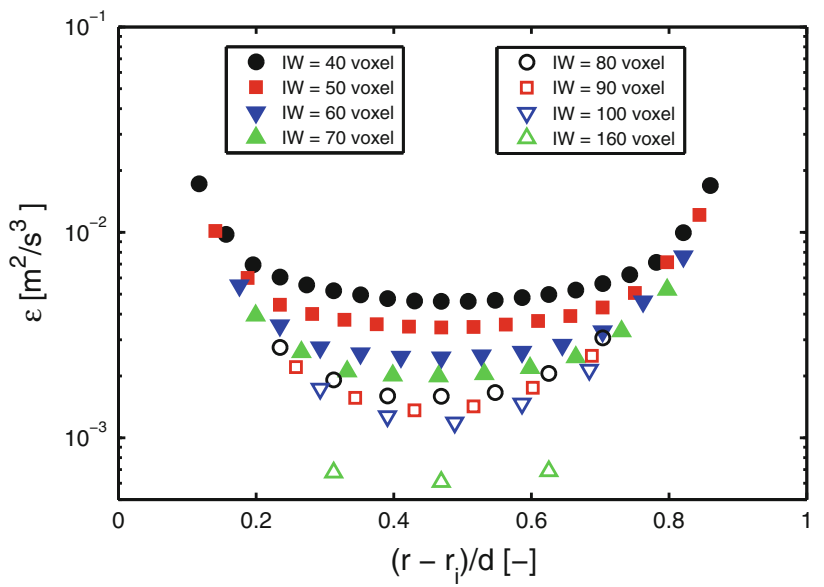

Fig. 20 Dissipation rate estimations for changing interrogation window sizes $(I W)$ for $R e_{s}=14,000$ with exact counter-rotation of the cylinders. $75 \%$ interrogation window overlapping was used for all interrogation domains. Spatial averaging was performed in the axial direction of the cylinders

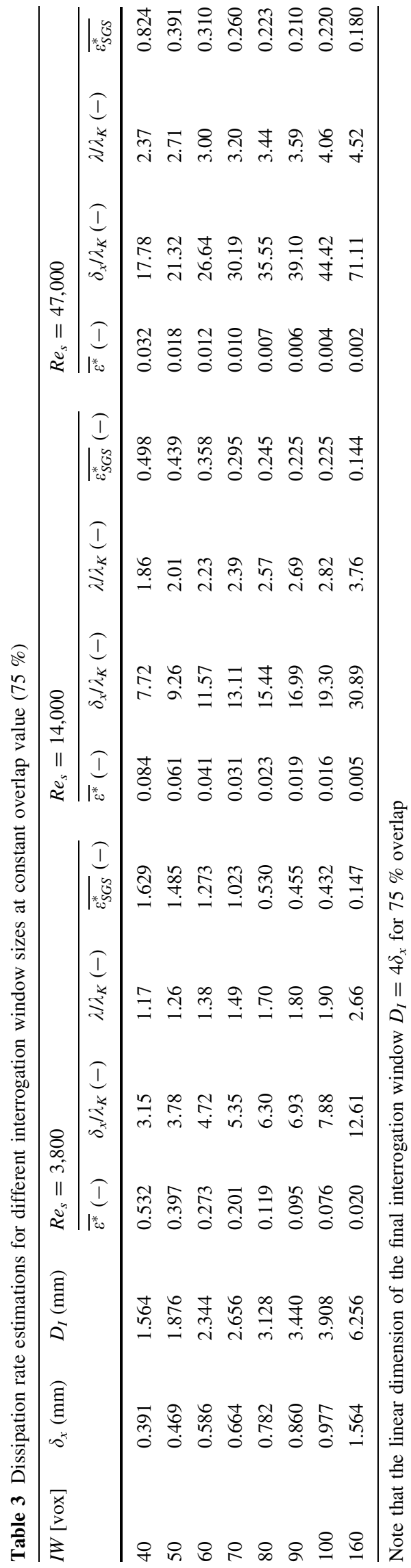


Table 4 Parameters that were used to compute the dissipation rates for the fully turbulent flow cases

\begin{tabular}{|c|c|c|c|c|}
\hline$R e_{s}(-)$ & $I W(\operatorname{vox})$ & Overlap (\%) & Kernel size $(-)$ & Number of excluded data points $(-)$ \\
\hline All & 40 & 75 & $5 \times 5 \times 5$ & 3 \\
\hline $3,800,14,000,47,000$ & 40 & 50 & $3 \times 3 \times 3$ & 2 \\
\hline $3,800,14,000,47,000$ & 40 & 0 & $1 \times 1 \times 1$ & 1 \\
\hline $3,800,14,000,47,000$ & $50,60, \ldots 100$ & 75 & $5 \times 5 \times 5$ & 3 \\
\hline $3,800,14,000,47,000$ & 80 & 50 & $3 \times 3 \times 3$ & 2 \\
\hline $3,800,14,000,47,000$ & 160 & 75 & $5 \times 5 \times 5$ & 2 \\
\hline
\end{tabular}

"Overlap" stands for interrogation window overlap value in percentage. "Kernel size" is the number of the neighbouring data points to calculate the velocity gradients during regression analysis. "Number of excluded data points" stands for the number of the data points excluded from the measurement domain at both inner and outer cylinder sides in the radial direction

The effect of the interrogation window size to the mean dissipation rate is summarised in Table 3. Consistent with previous reports in literature (Saarenrinne and Piirto 2000; Racina and Kind 2006; Tanaka and Eaton 2007; Lavoie et al. 2007), the estimations are decreasing with increasing size of the interrogation windows; $I W$ (i.e. with increasing values of $D_{I}$ and $\delta_{x}$ ), for all $R e_{s}$ numbers. If the values are compared to our best estimations (i.e. $I W=40$ voxel), doubling the interrogation window size results in an average decrease of $76 \%$ for all $R e_{s}$. The difference increases to an average of $95 \%$ in the case of 4-times larger window size $(I W=160$ voxel $)$.

\subsubsection{Effect of the overlap of the interrogation windows}

As the last approach, we discuss the effect of the overlap value of the final interrogation volumes to the dissipation rate estimations.

It should be noted that the velocity gradient computation scheme is the same for all cases in this study. However, for the computations in this section, we adjusted the kernel sizes according to the overlap values. The $5 \times 5 \times 5$ kernel size that is used to filter the measured velocity field is chosen equal to the interrogation window with $75 \%$ overlap (Elsinga et al. 2010). In order to match the kernel size to the correlation window size at 0 and $50 \%$ overlap, $1 \times 1 \times 1$ and $3 \times 3 \times 3$ kernel are used in these cases. This means that the velocity gradients for $0 \%$ overlap were calculated without any filtering. Thus, the expected noise level is higher for $0 \%$ overlap.

Since the kernel size is not constant, the number of excluded data points close to the cylinders are varied between the overlap values as well. Three, two and one data points were excluded from the measured domain for the analyses with 75,50 , and $0 \%$ overlap, respectively. The only exception to this procedure is the case of $160 \times 160 \times 160$ voxel $^{3}$ final interrogation window with $75 \%$ overlap. Since the measured data for this case contains only 7 points in the radial direction, three point exclusion results in the removal of 6 data points. Obviously, one data point in the radial direction is not enough for the total dissipation estimation and introduces additional uncertainty. Hence, we excluded two data points from the measurement domain at both sides for the velocity gradient estimations of the corresponding case. The parameters of the velocity gradient computations are summarised in Table 4.

Table 5 Dissipation rate estimations at a constant final interrogation window size ( $I W=40$ voxel, $\left.D_{I}=1.564 \mathrm{~mm}\right)$, but for different interrogation window overlap values

\begin{tabular}{lcllccccc}
\hline$R e_{s}(-)$ & Overlap $(\%)$ & $\delta_{x}(\mathrm{~mm})$ & $D_{I}(\mathrm{~mm})$ & $\delta_{x} / \lambda_{K}(-)$ & $D_{I} / \lambda_{K}(-)$ & $\overline{\varepsilon^{*}}(-)$ & $\lambda / \lambda_{K}(-)$ & $\overline{\varepsilon_{S G S}^{*}}(-)$ \\
\hline 3,800 & 0 & 1.564 & 1.564 & 12.61 & 12.61 & 0.200 & 1.50 \\
& 50 & 0.782 & 1.564 & 6.30 & 12.61 & 0.235 & 1.44 \\
& 75 & 0.391 & 1.564 & 3.15 & 12.61 & 0.532 & 1.17 \\
14,000 & 0 & 1.564 & 1.564 & 30.89 & 30.89 & 0.040 & 2.24 & 0.295 \\
& 50 & 0.782 & 1.564 & 15.44 & 30.89 & 0.046 & 2.16 & 0.139 \\
& 75 & 0.391 & 1.564 & 7.72 & 30.89 & 0.084 & 1.86 & 0.164 \\
47,000 & 0 & 1.564 & 1.564 & 71.11 & 71.11 & 0.012 & 3.04 & 0.118 \\
& 50 & 0.782 & 1.564 & 35.55 & 71.11 & 0.014 & 2.90 & 0.150 \\
& 75 & 0.391 & 1.564 & 17.78 & 71.11 & 0.032 & 2.37 & 0.824 \\
\hline
\end{tabular}


Table 6 Relation between interrogation window size, vector spacing and the dissipation rate estimations for $R e_{s}=14,000$

\begin{tabular}{|c|c|c|c|c|c|c|c|c|c|c|c|c|}
\hline \multirow[t]{2}{*}{$D_{I}(\mathrm{~mm})$} & \multicolumn{4}{|c|}{$\delta_{x}=0.391 \mathrm{~mm}$} & \multicolumn{4}{|c|}{$\delta_{x}=0.782 \mathrm{~mm}$} & \multicolumn{4}{|c|}{$\delta_{x}=1.564 \mathrm{~mm}$} \\
\hline & $\delta_{x} / \lambda_{K}(-)$ & $D_{I} / \lambda_{K}(-)$ & $\overline{\varepsilon^{*}}(-)$ & $\lambda / \lambda_{K}(-)$ & $\delta_{x} / \lambda_{K}(-)$ & $D_{I} / \lambda_{K}(-)$ & $\overline{\varepsilon^{*}}(-)$ & $\lambda / \lambda_{K}(-)$ & $\delta_{x} / \lambda_{K}(-)$ & $D_{I} / \lambda_{K}(-)$ & $\overline{\varepsilon^{*}}(-)$ & $\lambda / \lambda_{K}(-)$ \\
\hline 1.564 & 7.72 & 30.89 & 0.084 & 1.86 & 15.44 & 30.89 & 0.046 & 2.16 & 30.89 & 30.89 & 0.040 & 2.24 \\
\hline 3.128 & - & - & - & - & 15.44 & 61.78 & 0.023 & 2.57 & 30.89 & 61.78 & 0.012 & 3.00 \\
\hline 6.256 & - & - & - & - & - & - & - & - & 30.89 & 123.56 & 0.005 & 3.76 \\
\hline
\end{tabular}

$D_{I}=1.564,3.128$ and $6.256 \mathrm{~mm}$ correspond to $I W=40,80$ and 160 voxel, respectively. Moving horizontally from left to right in a row represents the decrease of the overlap values (from 75, 50 to $0 \%$ ), at a constant interrogation window size. For each row, the first non-empty cell from the left represents a $75 \%$ overlap value for the corresponding interrogation window size. Moving in the diagonal direction from top-left to bottom-right represents the increment of the interrogation window size at a constant overlap value. For instance; the cell for $D_{I}=3.128 \mathrm{~mm}$ and $\delta_{x}=1.564 \mathrm{~mm}$ stands for a $50 \%$ overlap, whereas the cell $D_{I}=6.256 \mathrm{~mm}$ and $\delta_{x}=1.564 \mathrm{~mm}$ represents a $75 \%$ overlap

The results of changing interrogation overlap values at a constant interrogation window size of $I W=40$ voxel are presented in Table 5. The dissipation rate estimations increase with increasing overlap value. Incrementing the overlap from 0 to 50 and $75 \%$, results in 15-17 and 55-60\% improvement of the estimation for all three values of $R e_{s}$ considered here.

The effect of overlap ratios was tested with $I W=80$ and 160 voxels as well. For the simplicity, results are given only for $R e_{s}=14,000$ in Table 6 . The performance of the dissipation rate estimations decreases for increasing vector space at a constant interrogation window size $D_{I}$. At a constant distance between vectors, $\delta_{x}$, increasing the interrogation window size results in lower dissipation estimations. However, the dependency of the estimations on the change of $D_{I}$ and $\delta_{x}$ are not the same. At a constant $D_{I}$, doubling $\delta_{x}$ results in better estimations than of doubling $D_{I}$ at a constant $\delta_{x}$.

\subsubsection{Dissipation rate estimations with large eddy method}

In order to estimate the contribution of the non-resolved scales to the dissipation rates, we computed the dissipation rates using the large eddy PIV (Sheng et al. 2000). Using the sub-grid scale (SGS) flux, the large eddy method takes the unresolved scales of PIV measurements into consideration for dissipation rate estimations (Sheng et al. 2000; Sharp and Adrian 2001).

The dissipation rate estimations by the large eddy PIV method were performed only for fully turbulent cases and are given by $\overline{\varepsilon_{S G S}^{*}}$ in Tables 2, 3 and 5, in normalised form. Similar to Sheng et al. (2000), we used $C_{S}=0.17$ as the Smagorinsky constant for the computations. As stated by Sheng et al. (2000), the Smagorinsky model results in better estimations for higher $R e_{s}$.

Our comparisons show that the large eddy method results in improved dissipation rate estimations than the direct estimations by tomographic PIV, as expected. The improvement for all cases indicates that the error in the direct estimation of dissipation rates by tomographic PIV is due to the unresolved scales.

\subsection{Summary of the dissipation rate estimations}

Our results are is summarised in Figs. 21 and 22. As reported in the literature, underestimation of the dissipation rates is evident for fully turbulent flow cases, and the degree of underestimation increases with Reynolds numbers (Sheng et al. 2000; Saarenrinne and Piirto 2000; Sharp and Adrian 2001; Racina and Kind 2006; Lavoie et al 2007; Tanaka and Eaton 2007).

The results show that the success of the dissipation rate estimations are strongly related to the spacing between the vectors and the interrogation window size. The error level increases with a logarithmic characteristic, as reported (Baldi and Yianneskis 2003; Racina and Kind 2006; Lavoie et al. 2007; Worth et al. 2010). Different cases with a constant overlap of $75 \%$ fall almost on same curve, with relatively small scatter. Decreasing window overlap at a constant interrogation window size increases the error.

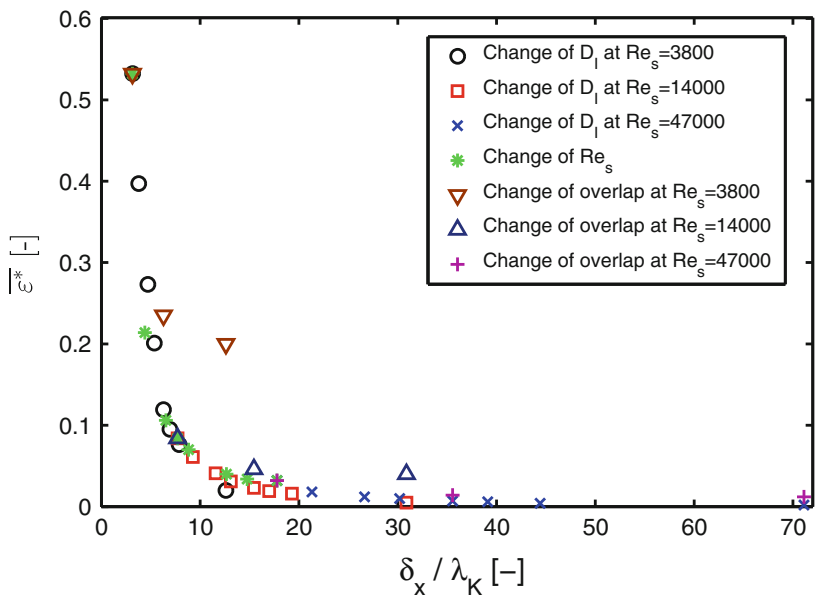

Fig. 21 Normalised mean dissipation rate estimations of three approaches for fully turbulent flow cases with exact counter-rotation. For details, see Tables 2, 3 and 5 


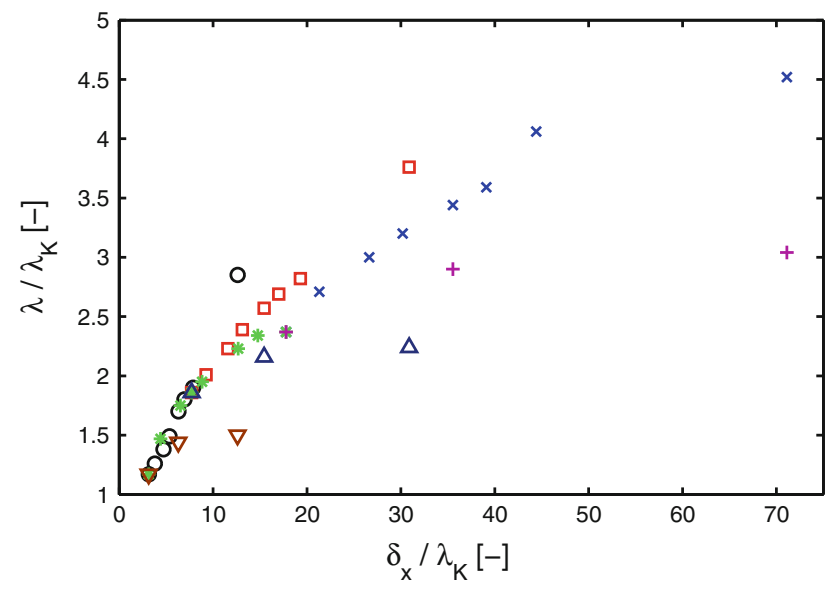

Fig. 22 Ratio of the equivalent Kolmogorov scale over the actual Kolmogorov scale $\left(\lambda / \lambda_{K}\right)$, as a function of the vector spacing relative to the Kolmogorov scale $\left(\delta_{x} / \lambda_{K}\right)$. The symbols follow the same coding as given in Fig. 21

However, it results in lower error levels compared to similar vector spacing with larger window sizes. Hence, the dissipation rate estimations, therefore the actual spatial resolution of tomographic PIV, is a non-linear function of both $\delta_{x} / \lambda_{K}$ and $D_{I} / \lambda_{K}$.

Extrapolating the values in Fig. 22 to $\lambda / \lambda_{K}=1$ implies that vector spacing of $\delta_{x} / \lambda_{K} \approx 1.5-2.0$ (equivalent to window sizes $D_{I} / \lambda_{K} \approx 6.0-8.0$ at $75 \%$ overlap or $D_{I} / \lambda_{K} \approx$ $3.0-4.0$ at $50 \%$ overlap) is required to fully resolve the turbulent dissipation scales. This is comparable with numbers reported by Buxton et al. (2011), Worth et al. (2010) and Saarenrinne and Piirto (2000) $\left(\delta_{x} / \lambda_{K}\right.$ $\approx 1.5-3.0$ ), which were calculated with $50 \%$ overlap. Our results are also seem consistent with a study by Jiménez et al. (1993), who reported an average diameter of Burgers' type vortices of $6-10 \lambda_{K}$.

In conclusion, the computations are more sensitive to changes of the interrogation window size than changes of the vector spacing. Although it results in a higher data density that possibly exceeds the tracer particle density, oversampling the measured data results in better estimations. The actual spatial sampling improves with the increasing window overlapping.

\section{Conclusion}

In this paper, we describe the implementation of tomographic PIV for a Taylor-Couette flow. This was achieved through a rotating and curved transparent outer wall, that is, without the usage of an enclosure to reduce the effects of refraction. We used fluorescent tracer particles, appropriate optical filters and black paint on the inside of the inner cylinder to reduce the effects of surface reflection of the incident laser light. The accuracy of the image calibration for this situation and the refinement through volumetric self-calibration was shown from the comparison of the measured velocity in the laminar flow state with the exact analytical solution.

An attractive feature of Taylor-Couette flow is that we could generate different flow conditions by changing the angular velocities of the inner and outer cylinders, that is, laminar flow, Taylor vortex flow, and turbulent flow. The laminar flow is stationary, while the Taylor-vortex flow is dominated by large-scale flow structures. The turbulent flow is without any dominant large-scale structures (i.e. "featureless" turbulence). We utilised this to determine the spatial resolution of tomographic PIV in relation to the length scales that are present in the flow. We use the measured torque on the cylinders to obtain an independent estimate of the dissipation rate and compare this with the dissipation rate as is estimated from the measured velocity gradients. In tomographic PIV, the velocity gradients can be measured of all three velocity components and for all three principal directions. Hence, it was possible to estimate the dissipation rate and Kolmogorov length scale without recourse to any symmetry assumptions.

As a metric to express the difference between the measured dissipation rate and the actual dissipation rate, we use the ratio of the dissipation rate as estimated from the PIV data relative to the dissipation rate given by the measured torque, as well as the ratio of the Kolmogorov scale determined from corresponding dissipation rates. Evidently, the accuracy is improved for decreasing dimensions of the (3D) interrogation window, while maintaining a minimum image density of at least 6 particle images per interrogation window. While the laminar flow and Taylor vortex flow cases appear to be fully resolved, the turbulent flow cases are increasingly under-resolved. Only the turbulent flow with the lowest Reynolds number investigated here appears to be (almost) fully resolved. The measured dissipation rate relative to the actual dissipation rate quickly drops with increasing Reynolds number. Yet, the use of the so-called large-eddy PIV method is capable to recover most of the dissipation due to unresolved scales. It should be noted that the large-eddy PIV method is valid when the sub-grid scales are sufficiently isotropic, which may not be always the case in the present study. Most notably, our results show that an increment of the overlap ratio for subsequent (3D) interrogation windows yield improved results. We also showed that a vector spacing in the range of $\delta_{x} / \lambda_{K} \approx 1.5-2.0$, which equals to $D_{I} / \lambda_{K} \approx 6.0-8.0$ at $75 \%$ overlap $\left(D_{I} / \lambda_{K} \approx\right.$ $3.0-4.0$ at $50 \%$ overlap), is required for a good estimation of turbulent dissipation with PIV.

It is noted that the actual accuracy is also determined by many data reduction steps and experimental parameters, such as the number of particle images inside the (3D) 
interrogation window, the smoothing of the measurement data or the velocity gradient computation method. They might change the contribution of the noise to the measurements. However, we showed that the contribution of the noise to our estimations is lower ( $3.4 \%$ maximum) compared to other error sources. Further investigation should be done to reveal the effect of different aspects in the data reduction in relation to achieving an improvement of accuracy by means of oversampling.

Acknowledgments This research was supported by FP7 Marie Curie Initial Training Network, ITN 215723, "Surface Physics for Advanced Manufacturing" project.

Open Access This article is distributed under the terms of the Creative Commons Attribution License which permits any use, distribution, and reproduction in any medium, provided the original author(s) and the source are credited.

\section{References}

Abcha N, Latrache N, Dumouchel F, Mutabazi I (2008) Qualitative relation between reflected light intensity by Kalliroscope flakes and velocity field in the Couette-Taylor flow system. Exp Fluids 45(1):85-94

Adrian RJ, Westerweel J (2011) Particle image velocimetry. Cambridge University Press, Cambridge

Akonur A, Lueptow RM (2003) Three-dimensional velocity field for wavy Taylor-Couette flow. Phys Fluids 15(4):947-960

Andereck CD, Liu SS, Swinney HL (1986) Flow regimes in a circular Couette system with independently rotating cylinders. J Fluid Mech 164:155-183

Baldi S, Yianneskis M (2003) On the direct measurement of turbulence energy dissipation in stirred vessels with PIV. Ind Eng Chem Res 42(26):7006-7016

Buxton ORH, Laizet S, Ganapathisubramani B (2011) The effects of resolution and noise on kinematic features of fine-scale turbulence. Exp Fluids 51(5):1417-1437

Cholemari MR (2007) Modeling and correction of peak-locking in digital PIV. Exp Fluids 42(6):913-922

Coles D (1965) Transition in circular Couette flow. J Fluid Mech 21(3):385-425

Coles D, Van Atta C (1966) Measured distortion of a laminar circular Couette flow by end effects. J Fluid Mech 25(3):513-521

Delfos R, Ravelet F, Westerweel J (2009) Scaling of torque in turbulent Taylor-Couette flow with background rotation. In: Eckhardt B (ed) Advances in turbulence XII, Springer, Berlin (Springer Proceedings in Physics 132:629-632)

Deng R, Arifin DY, Mak YC, Wang CH (2009) Characterization of Taylor vortex flow in a short liquid column. AIChE J 55(12):3056-3065

Di Prima RC, Swinney HL (1985) Instabilities and transition in flow between concentric rotating cylinders. In: Swinney HL, Gollub JP (eds) Hydrodynamic instabilities and the transition to turbulence, topics in applied physics, vol 45, chap 6, Springer, Berlin, pp 139-180

Dong S (2008) Turbulent flow between counter-rotating concentric cylinders: a direct numerical simulation study. J Fluid Mech 615:371-399

Donnelly RJ (1991) Taylor Couette flow: the early days. Phys Today 44(11):32-39
Dubrulle B, Dauchot O, Daviaud F, Longaretti PY, Richard D, Zahn JP (2005) Stability and turbulent transport in Taylor-Couette flow from analysis of experimental data. Phys Fluids 17(9):095103

Elsinga GE, Scarano F, Wieneke B, van Oudheusden BW (2006) Tomographic particle image velocimetry. Exp Fluids 41(6):933947

Elsinga GE, Adrian RJ, van Oudheusden BW, Scarano F (2010) Threedimensional vortex organization in a high-Reynolds-number supersonic turbulent boundary layer. J Fluid Mech 644:35-60

Elsinga GE, Westerweel J, Scarano F, Novara M (2011) On the velocity of ghost particles and the bias errors in TomographicPIV. Exp Fluids 50(4):825-838

Hunt JCR, Wray AA, Moin P (1988) Eddies, streams, and convergence zones in turbulent flows. In: Center for turbulence research proceedings of the summer program, CTR-S88, pp 193-208

Jiménez J, Wray AA, Saffman PG, Rogallo RS (1993) The structure of intense vorticity in isotropic turbulence. J Fluid Mech 255:65-90

Lavoie P, Avallone G, De Gregorio F, Romano GP, Antonia RA (2007) Spatial resolution of PIV for the measurement of turbulence. Exp Fluids 43(1):39-51

Lewis GS, Swinney HL (1999) Velocity structure functions, scaling, and transitions in high-Reynolds-number Couette-Taylor flow. Phys Rev E 59(5):5457-5467

McEligot DM, Nolan KP, Walsh EJ, Laurien E (2008) Effects of pressure gradients on entropy generation in the viscous layers of turbulent wall flows. Int J Heat Mass Transf 51(5-6):1104-1114

Racina A, Kind M (2006) Specific power input and local micromixing times in turbulent Taylor-Couette flow. Exp Fluids 41(3):513522

Ravelet F, Delfos R, Westerweel J (2010) Influence of global rotation and Reynolds number on the large-scale features of a turbulent Taylor-Couette flow. Phys Fluids 22(5):055103

Roth GI, Katz J (2001) Five techniques for increasing the speed and accuracy of PIV interrogation. Meas Sci Technol 12(3):238-245

Saarenrinne P, Piirto M (2000) Turbulent kinetic energy dissipation rate estimation from PIV velocity vector fields. Exp Fluids 29:300-307

Sharp KV, Adrian RJ (2001) PIV study of small-scale flow structure around a Rushton turbine. AIChE J 47(4):766-778

Sharp KV, Kim KC, Adrian RJ (2000) Dissipation estimation around a Rushton Turbine using Particle Image Velocimetry. In: Adrian RJ, Durao DFG, Durst F, Heitor MV, Maeda M, Whitelaw JH (eds) Laser techniques applied to fluid mechanics: selected papers from the 9th international symposium, Lisbon, Portugal, July 13-16, 1998, Springer, Berlin, pp 337-354

Sheng J, Meng H, Fox RO (2000) A large eddy PIV method for turbulence dissipation rate estimation. Chem Eng Sci 55(20):4423-4434

Smieszek M, Egbers C (2005) Flow structures and stability in Newtonian and non-Newtonian Taylor-Couette flow. J Phys Conf Ser 14:72-77

Tanaka T, Eaton JK (2007) A correction method for measuring turbulence kinetic energy dissipation rate by PIV. Exp Fluids 42(6):893-902

Vaezi V, Oh ES, Aldredge RC (1997) High-intensity turbulence measurements in a Taylor-Couette flow reactor. Exp Thermal Fluid Sci 15(4):424-431

Wang L, Olsen MG, Vigil RD (2005) Reappearance of azimuthal waves in turbulent Taylor Couette flow at large aspect ratio. Chem Eng Sci 60(20):5555-5568

Wereley ST, Lueptow RM (1998) Spatio-temporal character of nonwavy and wavy Taylor-Couette flow. J Fluid Mech 364:59-80

Wereley ST, Lueptow RM (1999) Velocity field for Taylor-Couette flow with an axial flow. Phys Fluids 11(12):3637-3649 
Westerweel J, Scarano F (2005) Universal outlier detection for PIV data. Exp Fluids 39(6): 1096-1100

Wieneke B (2008) Volume self-calibration for 3D particle image velocimetry. Exp Fluids 45(4):549-556
Worth NA, Nickels TB, Swaminathan N (2010) A tomographic PIV resolution study based on homogeneous isotropic turbulence DNS data. Exp Fluids 49(3):637-656 